NISSUNA UMANA INVESTIGAZIONE SI PUO DIMANDARE VERA SCIENZIA S'ESSA NON PASSA PER LE MATEMATICHE DIMOSTRAZIONI LEONARDO DA VINCI

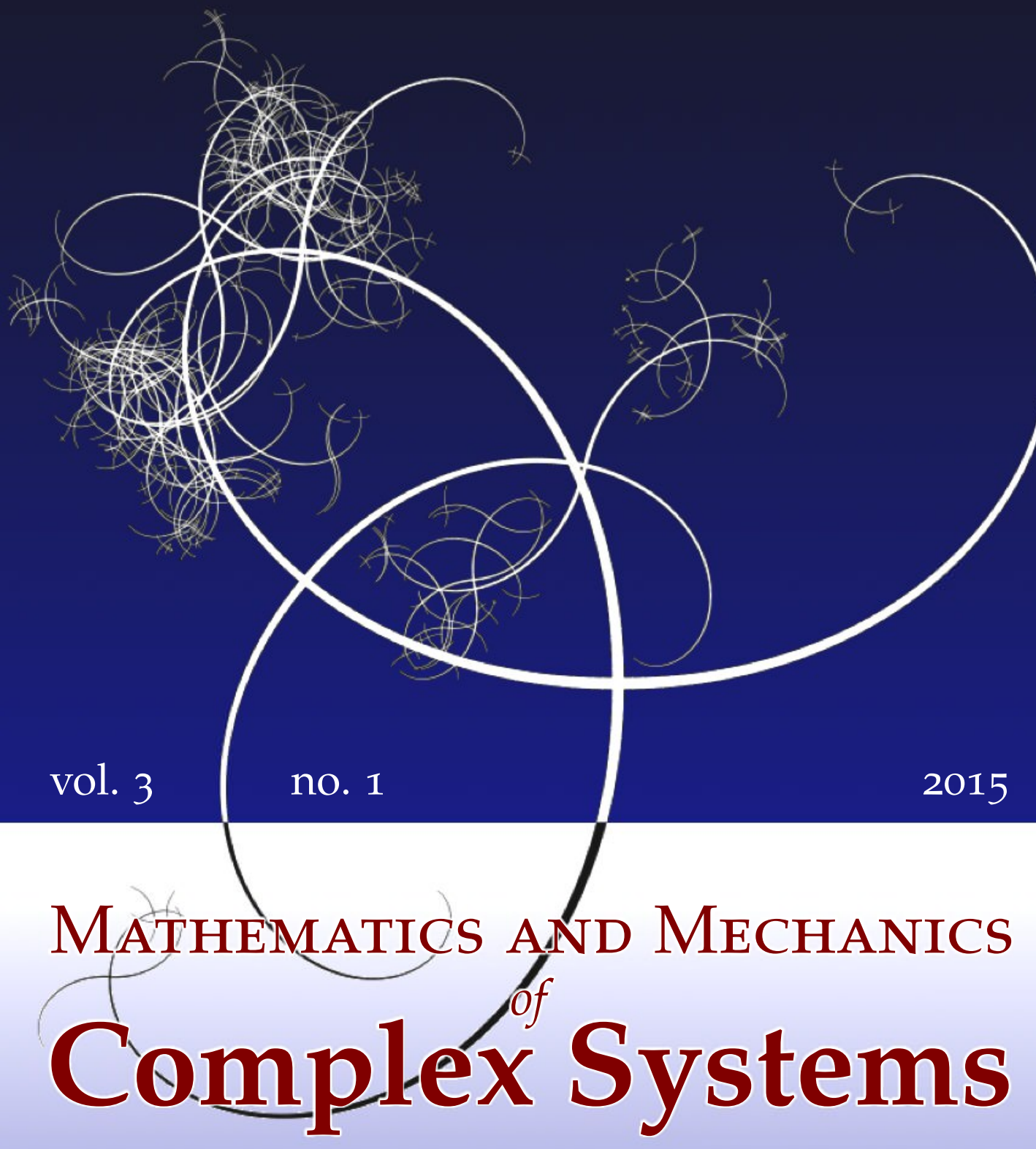

Angelo Luongo, Manuel Ferretti And Alexander P. Seyranian EFFECTS OF DAMPING ON THE STABILITY OF THE COMPRESSED NICOLAI BEAM 


\title{
EFFECTS OF DAMPING ON THE STABILITY OF THE COMPRESSED NICOLAI BEAM
}

\author{
Angelo Luongo, Manuel Ferretti and Alexander P. Seyranian
}

The Nicolai problem concerning the stability of a quasisymmetric cantilever beam embedded in a three-dimensional space, under a compressive dead load and a follower torque, is addressed. The effect of external and internal damping on stability is investigated. The partial differential equations of motion, accounting for the pretwist contribution, are recast in weak form via the Galerkin method, and a linear algebraic problem, governing the stability of the rectilinear configuration of the beam, is derived. Perturbation methods are used to analytically compute the eigenvalues, starting with an unperturbed, undamped, symmetric, untwisted beam, axially loaded, in both the subcritical and critical regimes. Accordingly, an asymmetry parameter, the torque, the damping, and the load increment are taken as perturbation parameters. Maclaurin series are used for semisimple eigenvalues occurring in subcritical states, and Puiseux series for the quadruple-zero eigenvalue existing at the Euler point. Based on the eigenvalue behavior described by the asymptotic expansions, the stability domains are constructed in the two or three-dimensional space of the bifurcation parameters. It is found that dynamic bifurcations occur in the subcritical regime, and dynamic or static bifurcations in the critical regime. It is shown that stability is governed mostly by the bifurcation of the lowest eigenvalue. In all cases the Nicolai paradox is recovered, and the beneficial effects of asymmetry and damping are highlighted.

\section{Introduction}

The fascinating mechanical problem first formulated by Nicolai [1928] and now bearing his name consists in determining the critical value of a follower (tangential) torque acting at the free end of a uniform elastic cantilever beam embedded in a three-dimensional space, with equal moments of inertia in the two planes. The Nicolai paradox consists in the fact that the bifurcation value of the torque is zero, in the sense that a vanishingly small torque is able to cause (dynamic) instability

\section{Communicated by Antonio Carcaterra.}

MSC2010: 74H10, 74H55, 74H60.

Keywords: stability of beams, nonconservative system, Nicolai paradox, perturbation methods, semisimple eigenvalues, quadruple-zero eigenvalue. 
of the beam. Nicolai found this (apparently) surprising result by using a simplified two-degree of freedom model with lumped mass; he also found that damping is stabilizing. Subsequently he analyzed the effects of small asymmetries in the two inertia moments, and found they have a beneficial effect on stability [Nicolai 1929].

The paradox has recently been explained in [Seyranian and Mailybaev 2011], where it has been shown to be due to the bifurcation of a double semisimple eigenvalue, which leads to a stability domain with a conic singularity at the origin of parameter space, where the ideal symmetric system is located. Therefore, an infinitesimal perturbation of this state can lead the system out of the cone, causing instability. In the same paper the authors, by referring to a general discrete system, briefly investigated damping effects, arriving at a justification of the findings of Nicolai. In their paper, however, although they accounted for the presence of an axial load, they assumed it as below the Euler critical value.

In [Seyranian et al. 2014], the continuous model was enriched by accounting for the pretwist generated by the torque in the reference configuration. This effect, which had been neglected in previous papers, was, however, found to not affect the stability domain. The authors, by using analytical and numerical methods, also studied the neighborhood of the Euler point, although they did not address the (complex) mechanism of bifurcation. Moreover, they did not account for damping.

In this paper we reconsider the problem of Nicolai using a continuous model of a beam with pretwisting and introducing damping forces. We mainly focus on the influence of damping on the stability domains of the system. Thus, the problem of Nicolai is studied in greater depth, with multiple aims, namely: to thoroughly analyze the effects on stability of external and internal damping acting on the beam; to study the regimes of subcritical and critical axial loads and their influence on the critical value of the torque; to investigate the role of eigenvalues higher than the first in affecting stability; and to explain, by analytical methods, the mechanism of the bifurcation of the quadruple-zero eigenvalue which occurs at the Euler critical load. All these aspects are believed to be new; the latter, moreover, could have value that transcends the issue at hand.

The paper is organized as follows. In Section 2 the equations of motion are recalled, a Galerkin reduction is carried out, and a linear eigenvalue problem is drawn. In Section 3 the stability problem for subcritically loaded beams is addressed by performing a perturbation of semisimple eigenvalues. In Section 4 the stability problem for nearly critically loaded beams is tackled. Here it is shown that a Puiseux series expansion must be used to analyze bifurcation of the quadruple nonsemisimple eigenvalue. Differences in the algorithms for undamped and damped systems are also extensively commented upon for this occurrence. In all cases two or threedimensional stability domains are constructed and the type of bifurcation (static or dynamic) occurring at the different branches of the boundaries is commented upon. 


\section{Problem formulation}

Continuous model. We consider a cantilever beam, of length $l$ and mass per unit length $m$, loaded at the free end by a compressive axial dead load $P$ and a follower torque $L$ (see figure on the right). The system is assumed to be "nearly symmetric", in the sense that its geometric characteristics in the two principal planes are almost equal. The goal of this analysis is to evaluate the critical value of the torque at which the beam loses stability, by accounting for asymmetries, axial load, and damping.

The equations of motion for the elastic beam, modeled ac-

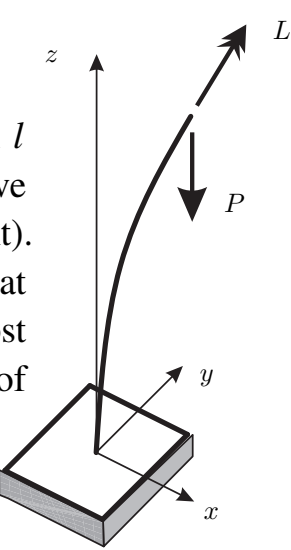
cording to the Euler-Bernoulli hypotheses, were derived in [Bolotin 1963]; in [Seyranian et al. 2014] the effect of the pretwist induced by the torque was included in the analysis. In this paper we consider a further improved model by accounting for internal and external dampings. The relevant equations of motion (see Appendix $\mathrm{C}$ for derivation) are

$$
\begin{aligned}
& m \ddot{u}+E I_{y} u^{I V}+L\left(1-2 \frac{E}{G} \frac{I_{x}+I_{y}}{J}\right) v^{\prime \prime \prime}+P u^{\prime \prime}-2 P \frac{L}{G J} v^{\prime}+\xi \dot{u}+\eta I_{y} \dot{u}^{I V}=0, \\
& m \ddot{v}+E I_{x} v^{I V}-L\left(1-2 \frac{E}{G} \frac{I_{x}+I_{y}}{J}\right) u^{\prime \prime \prime}+P v^{\prime \prime}+2 P \frac{L}{G J} u^{\prime}+\xi \dot{v}+\eta I_{x} \dot{v}^{I V}=0,
\end{aligned}
$$

with the boundary conditions

$$
\begin{aligned}
u(0) & =u^{\prime}(0)=0, \quad E I_{y} u^{\prime \prime}(l)+\eta I_{y} \dot{u}^{\prime \prime}(l)-2 L \frac{E I_{y}}{G J} v^{\prime}(l)=0, \\
v(0) & =v^{\prime}(0)=0, \quad E I_{x} v^{\prime \prime}(l)+\eta I_{x} \dot{v}^{\prime \prime}(l)+2 L \frac{E I_{x}}{G J} u^{\prime}(l)=0, \\
& -E I_{y} u^{\prime \prime \prime}(l)-\eta I_{y} \dot{u}^{\prime \prime \prime}(l)-P\left(u^{\prime}(l)-\frac{L}{G J} v(l)\right)=0, \\
& -E I_{x} v^{\prime \prime \prime}(l)-\eta I_{x} \dot{v}^{\prime \prime \prime}(l)-P\left(v^{\prime}(l)+\frac{L}{G J} u(l)\right)=0 .
\end{aligned}
$$

Here $u(z, t)$ and $v(z, t)$ are the transverse displacements of the centroid at the abscissa $z$ and time $t$, along the principal $x$ and $y$ axes, respectively; $E$ is the Young modulus of the material; $I_{x}$ and $I_{y}$ are the principal inertia moments of the cross-section and $J$ is its torsional inertia moment; $\xi$ and $\eta$ are the external damping and viscosity coefficients, respectively; a dash denotes differentiation with respect to $z$; and a dot denotes differentiation with respect to $t$. When the damping coefficients are set to zero, the equations studied in [Seyranian et al. 2014] are recovered. These equations govern the small oscillations of the pretwisted beam around the rectilinear configuration. 
The following nondimensional quantities are introduced:

$$
\begin{gathered}
\tilde{z}:=\frac{z}{l}, \quad \tilde{u}:=\frac{u}{l}, \quad \tilde{v}:=\frac{v}{l}, \quad \beta:=2 \frac{E}{G} \frac{I_{x}+I_{y}}{J}, \quad \gamma:=2 \frac{E}{G} \frac{I_{0}}{J}, \\
\tilde{L}:=\frac{L l}{E I_{0}}, \quad \tilde{P}:=\frac{P l^{2}}{E I_{0}}, \quad \tilde{I}_{y}:=\frac{I_{y}}{I_{0}}, \quad \tilde{I}_{x}:=\frac{I_{x}}{I_{0}}, \quad \tilde{m}:=\frac{m}{m_{0}}, \\
\tilde{t}:=t \sqrt{\frac{E I_{0}}{m_{0} l^{4}}}, \quad \tilde{\xi}:=\xi \sqrt{\frac{l^{4}}{m_{0} E I_{0}}}, \quad \tilde{\eta}:=\eta \sqrt{\frac{I_{0}}{m_{0} l^{4} E}},
\end{gathered}
$$

where $I_{0}$ and $m_{0}$ are an inertia moment and mass per unit length, respectively, taken as characteristics of a "close" ideal symmetric system, from which the actual system can be generated via a small perturbation (see Appendix A). With definitions (3), the equations of motion transform into

$$
\begin{aligned}
& m \ddot{u}+I_{y} u^{I V}+L(1-\beta) v^{\prime \prime \prime}+P u^{\prime \prime}-P L \gamma v^{\prime}+\xi \dot{u}+\eta I_{y} \dot{u}^{I V}=0, \\
& m \ddot{v}+I_{x} v^{I V}-L(1-\beta) u^{\prime \prime \prime}+P v^{\prime \prime}+P L \gamma u^{\prime}+\xi \dot{v}+\eta I_{x} \dot{v}^{I V}=0,
\end{aligned}
$$

and the boundary conditions into

$$
\begin{gathered}
u(0)=u^{\prime}(0)=0, \quad I_{y} u^{\prime \prime}(1)+\eta I_{y} \dot{u}^{\prime \prime}(1)-\gamma L I_{y} v^{\prime}(1)=0, \\
v(0)=v^{\prime}(0)=0, \quad I_{x} v^{\prime \prime}(1)+\eta I_{x} \dot{v}^{\prime \prime}(1)+\gamma L I_{x} u^{\prime}(1)=0, \\
-I_{y} u^{\prime \prime \prime}(1)-\eta I_{y} \dot{u}^{\prime \prime \prime}(1)-P\left(u^{\prime}(1)-\frac{1}{2} L \gamma v(1)\right)=0, \\
-I_{x} v^{\prime \prime \prime}(1)-\eta I_{x} \dot{v}^{\prime \prime \prime}(1)-P\left(v^{\prime}(1)+\frac{1}{2} L \gamma u(1)\right)=0,
\end{gathered}
$$

where the tilde has been suppressed for notational convenience.

Discrete model. A weak form of the problem (4), (5) is derived according to the weighted residuals (or extended Galerkin [Leipholz 1974; Zienkiewicz et al. 2005]) method. The unknown displacement fields are expressed as linear combinations of $2 N$ unknown time-dependent amplitudes, $\boldsymbol{x}:=\left(x_{i}(t)\right)^{T}$ and $\boldsymbol{y}:=\left(y_{i}(t)\right)^{T}$, and $N$ known space-dependent trial functions $\phi:=\left(\phi_{i}(z)\right)^{T}$, namely

$$
\begin{aligned}
& u(z, t)=\boldsymbol{\phi}^{T} \boldsymbol{x}, \\
& v(z, t)=\boldsymbol{\phi}^{T} \boldsymbol{y},
\end{aligned}
$$

where $\phi_{i}(0)=\phi_{i}^{\prime}(0)=0$ satisfy the geometrical boundary conditions; however, they are not required to satisfy the mechanical boundary conditions [Leipholz 1974; Zienkiewicz et al. 2005].

By substituting (6) into the field equations (4) and boundary conditions (5), residuals in the domain and at the free end $z=1$ are found, which are required to be orthogonal to the trial function itself: 


$$
\begin{aligned}
\sum_{i=1}^{N}\left\{\int _ { 0 } ^ { 1 } \phi _ { j } \left[m \phi_{i} \ddot{x}_{i}+I_{y} \phi_{i}^{I V} x_{i}+L(1-\beta) \phi_{i}^{\prime \prime \prime} y_{i}+P \phi_{i}^{\prime \prime} x_{i}-P L \gamma \phi_{i}^{\prime} y_{i}\right.\right. \\
\left.+\xi \phi_{i} \dot{x}_{i}+\eta I_{y} \phi_{i}^{I V} \dot{x}_{i}\right] d z+\phi_{j}^{\prime}\left[I_{y} \phi_{i}^{\prime \prime} x_{i}+\eta I_{y} \phi_{i}^{\prime \prime} \dot{x}_{i}-\gamma L I_{y} \phi_{i}^{\prime} y_{i}\right]_{z=1} \\
\left.+\phi_{j}\left[-I_{y} \phi_{i}^{\prime \prime \prime} x_{i}-\eta I_{y} \phi_{i}^{\prime \prime \prime} \dot{x}_{i}-P \phi_{i}^{\prime} x_{i}+\frac{P L \gamma}{2} \phi_{i} y_{i}\right]_{z=1}\right\}=0 \\
\sum_{i=1}^{N}\left\{\int _ { 0 } ^ { 1 } \phi _ { j } \left[m \phi_{i} \ddot{y}_{i}+I_{x} \phi_{i}^{I V} y_{i}-L(1-\beta) \phi_{i}^{\prime \prime \prime} x_{i}+P \phi_{i}^{\prime \prime} y_{i}+P L \gamma \phi_{i}^{\prime} x_{i}\right.\right. \\
\left.+\xi \phi_{i} \dot{y}_{i}+\eta I_{x} \phi_{i}^{I V} \dot{y}_{i}\right] d z+\phi_{j}^{\prime}\left[I_{x} \phi_{i}^{\prime \prime} y_{i}+\eta I_{x} \phi_{i}^{\prime \prime} \dot{y}_{i}+\gamma L I_{x} \phi_{i}^{\prime} x_{i}\right]_{z=1} \\
\left.+\phi_{j}\left[-I_{x} \phi_{i}^{\prime \prime \prime} y_{i}-\eta I_{x} \phi_{i}^{\prime \prime \prime} \dot{y}_{i}-P \phi_{i}^{\prime} y_{i}-\frac{P L \gamma}{2} \phi_{i} x_{i}\right]_{z=1}\right\}=0,
\end{aligned}
$$

where $j=1,2, \ldots, N$. After integration by parts and accounting for the geometric boundary conditions, all terms at the boundaries disappear. From these, a set of $2 \mathrm{~N}$ ordinary differential equations is derived:

$$
\boldsymbol{M} \ddot{\boldsymbol{q}}+\boldsymbol{C} \dot{\boldsymbol{q}}+(\boldsymbol{K}+\boldsymbol{H}) \boldsymbol{q}=\mathbf{0},
$$

where $\boldsymbol{q}:=(\boldsymbol{x}, \boldsymbol{y})^{T}$ is a $2 N$ column vector of the unknown amplitudes, and $\boldsymbol{M}$ is the mass, $\boldsymbol{C}$ the damping, $\boldsymbol{K}$ the stiffness, and $\boldsymbol{H}$ the circulatory matrices, all of dimension $2 N \times 2 N$, defined by

$$
\begin{array}{rlrl}
\boldsymbol{C} & :=\left[\begin{array}{cc}
\xi \boldsymbol{m}+\eta I_{y} \boldsymbol{k}_{E} & \mathbf{0} \\
\mathbf{0} & \xi \boldsymbol{m}+\eta I_{x} \boldsymbol{k}_{E}
\end{array}\right], & \boldsymbol{M}:=m\left[\begin{array}{cc}
\boldsymbol{m} & \mathbf{0} \\
\mathbf{0} & \boldsymbol{m}
\end{array}\right], \\
\boldsymbol{K} & :=\left[\begin{array}{cc}
I_{y} \boldsymbol{k}_{E}+P \boldsymbol{k}_{G} & \mathbf{0} \\
\mathbf{0} & I_{x} \boldsymbol{k}_{E}+P \boldsymbol{k}_{G}
\end{array}\right], & \boldsymbol{H}_{u}:=\left[\begin{array}{cc}
\mathbf{0} & \boldsymbol{h}_{1} \\
-\boldsymbol{h}_{1} & \mathbf{0}
\end{array}\right], \\
\boldsymbol{H}_{t}:=\left[\begin{array}{ccc}
0 & -\beta \boldsymbol{h}_{1}-P \gamma \boldsymbol{h}_{2}-\gamma I_{y} \boldsymbol{h}_{3} \\
\beta \boldsymbol{h}_{1}+P \gamma \boldsymbol{h}_{2}+\gamma I_{x} \boldsymbol{h}_{3} & \mathbf{0}
\end{array}\right], & \boldsymbol{H}:=L\left(\boldsymbol{H}_{u}+\boldsymbol{H}_{t}\right) .
\end{array}
$$

In these equations, the following $N \times N$ submatrices appear, which depend on the trial functions only:

$$
\begin{array}{rlrl}
\boldsymbol{m} & =\int_{0}^{1} \boldsymbol{\phi} \boldsymbol{\phi}^{T} d z, & \boldsymbol{k}_{E}=\int_{0}^{1} \boldsymbol{\phi}^{\prime \prime} \boldsymbol{\phi}^{\prime \prime T} d z, \\
\boldsymbol{k}_{G}=-\int_{0}^{1} \boldsymbol{\phi}^{\prime} \boldsymbol{\phi}^{\prime T} d z, & \boldsymbol{h}_{1}=\int_{0}^{1} \boldsymbol{\phi} \boldsymbol{\phi}^{\prime \prime \prime} T d z \\
\boldsymbol{h}_{2}=\int_{0}^{1} \boldsymbol{\phi} \boldsymbol{\phi}^{\prime T} d z-\left.\frac{1}{2} \boldsymbol{\phi} \boldsymbol{\phi}^{T}\right|_{z=1}, & \boldsymbol{h}_{3}=\left.\boldsymbol{\phi}^{\prime} \boldsymbol{\phi}^{\prime T}\right|_{z=1},
\end{array}
$$

where the indices $E$ and $G$ refer to the elastic and geometric parts of the stiffness matrix and the indices $u$ and $t$ refer to the untwisted and twisted beams, that is, to the torsionally rigid or torsionally elastic beams. Notice that the coupling between the $\boldsymbol{x}$ and $\boldsymbol{y}$ variables is exclusively due to the torque. 
In the numerical simulations to be performed ahead, we will take as trial functions the (mutually orthogonal) eigenfunctions of the free undamped oscillations of the unprestressed planar cantilever (see Appendix B). Due to their orthogonality properties and normalization, it follows that $\boldsymbol{m}=\boldsymbol{I}$ and $\boldsymbol{k}_{E}=\operatorname{diag}\left(\omega_{i}^{2}\right)$, where $\omega_{i}$ are the (nondimensional) natural frequencies; in contrast, $\boldsymbol{k}_{G}$ and $\boldsymbol{h}_{i}, i=1, \ldots, 3$, are full matrices. Moreover, while $\boldsymbol{M}, \boldsymbol{C}$, and $\boldsymbol{K}$ are symmetric matrices, $\boldsymbol{H}$ is not symmetric nor antisymmetric. Finally, the damping submatrices are linear combinations of the mass and elastic stiffness submatrices, as in the Rayleigh model of damping.

The algebraic eigenvalue problem. Substitution of $\boldsymbol{q}(t)=\boldsymbol{w} e^{\lambda t}$ in (8) and premultiplication by $\boldsymbol{M}^{-1}$ leads to the algebraic eigenvalue problem

$$
\left(\boldsymbol{A}+\lambda \boldsymbol{D}+\lambda^{2} \boldsymbol{I}\right) \boldsymbol{w}=\mathbf{0},
$$

in which $\boldsymbol{A}:=\boldsymbol{M}^{-1}(\boldsymbol{K}+\boldsymbol{H})$ and $\boldsymbol{D}:=\boldsymbol{M}^{-1} \boldsymbol{C}$. The trivial equilibrium is (asymptotically) stable if $\operatorname{Re} \lambda<0$ for all $\lambda$, and is unstable if $\operatorname{Re} \lambda>0$ for at least one $\lambda$.

When the system is undamped (that is, $\boldsymbol{D}=\mathbf{0}$ ), the eigenvalue problem is more conveniently recast in the standard form:

$$
(\boldsymbol{A}-\mu \boldsymbol{I}) \boldsymbol{w}=\mathbf{0},
$$

where $\mu:=-\lambda^{2}$. The trivial equilibrium is stable if all $\mu$ are real and positive (that is, $\lambda$ is purely imaginary), and it is unstable if at least one $\mu$ is negative or complex (entailing that one root $\lambda$ has a positive real part).

\section{Stability analysis for subcritically compressed beams}

The perturbed eigenvalue problem. We address the stability problem for the case in which the (nondimensional) axial load $P$ is lower than the (nondimensional) Eulerian critical load $P_{E}:=\pi^{2} / 4$. We assume that the cross-section is nearly symmetric, affected by a small asymmetry parameter $\alpha$; both the internal, $\eta$, and external, $\xi$, damping coefficients are small; and the follower torque, $L$, is also small. Accordingly, we introduce the following parameter rescaling:

$$
(\alpha, L, \xi, \eta) \rightarrow \varepsilon(\alpha, L, \xi, \eta),
$$

where $0<\varepsilon \ll 1$ is a perturbation parameter (artificially introduced, and to be reabsorbed at the end of the procedure). The (nondimensional) geometric characteristics and the mass per unit length (with a proper choice of $I_{0}$ and $m_{0}$ appearing in (3)), after series expansion, read (see Appendix A for an example)

$$
\left(\begin{array}{l}
I_{x} \\
I_{y} \\
m
\end{array}\right)=\left(\begin{array}{l}
1 \\
1 \\
1
\end{array}\right)+\varepsilon \alpha\left(\begin{array}{c}
I_{x_{1}} \\
I_{y_{1}} \\
m_{1}
\end{array}\right)+O\left(\varepsilon^{2}\right)
$$


moreover, $\beta=\beta_{0}+O(\varepsilon)$ and $\gamma=\gamma_{0}+O(\varepsilon)$, with $\beta_{0}=2 \gamma_{0}$. Consequently, the matrices in (11) can also be expressed in series form as

$$
\boldsymbol{A}=\boldsymbol{A}_{0}+\varepsilon \boldsymbol{A}_{1}+O\left(\varepsilon^{2}\right), \quad \boldsymbol{D}=\varepsilon \boldsymbol{D}_{1}+O\left(\varepsilon^{2}\right),
$$

where

$$
\begin{aligned}
\boldsymbol{A}_{0} & =\left[\begin{array}{cc}
\boldsymbol{k}_{E}+P \boldsymbol{k}_{G} & \mathbf{0} \\
\mathbf{0} & \boldsymbol{k}_{E}+P \boldsymbol{k}_{G}
\end{array}\right], \\
\boldsymbol{A}_{1} & =\boldsymbol{A}_{1_{u}}+\boldsymbol{A}_{1_{t}}, \\
\boldsymbol{A}_{1_{u}} & =\left[\begin{array}{cc}
\alpha\left(I_{y 1}-m_{1}\right) \boldsymbol{k}_{E}-\alpha m_{1} P \boldsymbol{k}_{G} & L \boldsymbol{h}_{1} \\
-L \boldsymbol{h}_{1} & \alpha\left(I_{x 1}-m_{1}\right) \boldsymbol{k}_{E}-\alpha m_{1} P \boldsymbol{k}_{G}
\end{array}\right] \\
\boldsymbol{A}_{1_{t}} & =\left[\begin{array}{cc}
L\left(\beta_{0} \boldsymbol{h}_{1}+P \gamma_{0} \boldsymbol{h}_{2}+\gamma_{0} \boldsymbol{h}_{3}\right) & -L\left(\beta_{0} \boldsymbol{h}_{1}+P \gamma_{0} \boldsymbol{h}_{2}+\gamma_{0} \boldsymbol{h}_{3}\right) \\
\mathbf{0} & \mathbf{0}
\end{array}\right], \\
\boldsymbol{D}_{1}= & {\left[\begin{array}{cc}
\xi \boldsymbol{m}+\eta \boldsymbol{k}_{E} & \mathbf{0} \\
\mathbf{0} & \xi \boldsymbol{m}+\eta \boldsymbol{k}_{E}
\end{array}\right] . }
\end{aligned}
$$

The eigenvalue problem (11), therefore, appears as a perturbation of the problem relevant to the symmetric, undamped, subcritically prestressed beam, with no torque, namely:

$$
\left[\boldsymbol{A}_{0}+\lambda^{2} \boldsymbol{I}+\varepsilon\left(\boldsymbol{A}_{1}+\lambda \boldsymbol{D}_{1}\right)+\cdots\right] \boldsymbol{w}=\mathbf{0},
$$

where $\boldsymbol{A}_{1}$ accounts for (first-order) asymmetry and torque, while $\boldsymbol{D}_{1}$ accounts for damping.

\section{Perturbation analysis.}

The damped case. The eigenvalue problem (17) is solved by a perturbation method. Due to the symmetry of the unperturbed mechanical system, the eigenvalue $\lambda_{0}$ is a semisimple eigenvalue for the matrix $\boldsymbol{A}_{0}$, that is, two independent eigenvectors are associated with any eigenvalue, each representing a mode of oscillation in the $(x, z)$-plane or the $(y, z)$-plane. Such an eigenvalue and its associated eigenvectors admit Maclaurin series expansion [Seyranian and Mailybaev 2003]:

$$
\lambda=\lambda_{0}+\varepsilon \lambda_{1}+\cdots, \quad \boldsymbol{w}=\boldsymbol{w}_{0}+\varepsilon \boldsymbol{w}_{1}+\cdots .
$$

By introducing (18) in the eigenvalue problem (17) and separately equating to zero terms with the same powers of $\varepsilon$, the following perturbation equations are obtained:

$$
\begin{aligned}
& \varepsilon^{0}:\left(\boldsymbol{A}_{0}+\lambda_{0}^{2} \boldsymbol{I}\right) \boldsymbol{w}_{0}=\mathbf{0}, \\
& \varepsilon^{1}:\left(\boldsymbol{A}_{0}+\lambda_{0}^{2} \boldsymbol{I}\right) \boldsymbol{w}_{1}=-\left(\boldsymbol{A}_{1}+\lambda_{0} \boldsymbol{D}_{1}+2 \lambda_{0} \lambda_{1} \boldsymbol{I}\right) \boldsymbol{w}_{0} .
\end{aligned}
$$


Since $\boldsymbol{A}_{0}$ is symmetric and positive definite, its eigenvalues $\lambda_{0}$ are pairs of complex conjugate purely imaginary numbers, that is, $\pm i \omega_{k}, k=1,2, \ldots, N$. The associated eigenvectors are real, and right and left eigenvectors coincide. We take $\lambda_{0}=+i \omega_{k}$, and denote by $\boldsymbol{u}_{1}$ and $\boldsymbol{u}_{2}$ the associated eigenvectors, which are mutually orthogonal and normalized, that is, $\boldsymbol{u}_{i}^{T} \boldsymbol{u}_{j}=\delta_{i j}$. Hence, the $\varepsilon^{0}$-order perturbation equation admits the general solution

$$
\boldsymbol{w}_{0}=\boldsymbol{U} \boldsymbol{a},
$$

where $\boldsymbol{U}=\left(\boldsymbol{u}_{1}, \boldsymbol{u}_{2}\right)$ is a $2 N \times 2$ modal matrix and $\boldsymbol{a}=\left(a_{1}, a_{2}\right)^{T}$ is a column vector listing two unknown amplitudes. Note that, at this order, any linear combination of $\boldsymbol{u}_{1}$ and $\boldsymbol{u}_{2}$ is an eigenvector, the indeterminacy being resolved only at the next order.

With (20), the $\varepsilon$-order equation reads

$$
\left(\boldsymbol{A}_{0}+\lambda_{0}^{2} \boldsymbol{I}\right) \boldsymbol{w}_{1}=-\left(\boldsymbol{A}_{1}+\lambda_{0} \boldsymbol{D}_{1}+2 \lambda_{0} \lambda_{1} \boldsymbol{I}\right) \boldsymbol{U} \boldsymbol{a} .
$$

This is a nonhomogeneous problem in which the linear operator $\boldsymbol{A}_{0}+\lambda_{0}^{2} \boldsymbol{I}$ is singular. In order to solve it, the right-hand member must be orthogonal to the kernel of the adjoint operator (the compatibility condition). Since this space is spanned by the rows of $\boldsymbol{U}^{T}$, the compatibility reads

$$
\left(\hat{\boldsymbol{A}}_{1}+\lambda_{0} \hat{\boldsymbol{D}}_{1}+2 \lambda_{0} \lambda_{1} \boldsymbol{I}\right) \boldsymbol{a}=\mathbf{0},
$$

where

$$
\hat{\boldsymbol{A}}_{1}:=\boldsymbol{U}^{T} \boldsymbol{A}_{1} \boldsymbol{U} \text { and } \quad \hat{\boldsymbol{D}}_{1}:=\boldsymbol{U}^{T} \boldsymbol{D}_{1} \boldsymbol{U}
$$

are $2 \times 2$ matrices representing the restrictions of $\boldsymbol{A}_{1}$ and $\boldsymbol{D}_{1}$ to the plane spanned by the columns of $\boldsymbol{U}$; moreover, $\boldsymbol{U}^{T} \boldsymbol{U}=\boldsymbol{I}$ has been used, which follows from the orthonormalization properties of the eigenvectors. A remarkable result is that the matrix $\hat{\boldsymbol{A}}_{1}$ does not depend on the pretwist, since the restriction of the matrix $\boldsymbol{A}_{1_{t}}$ to the plane of the eigenvectors is zero (see Appendix D for details). Therefore, to first order, stability is unaffected by the pretwist.

Equation (22) is an eigenvalue problem in the $\lambda_{1}$ unknown. The relevant characteristic equation is

$$
\lambda_{1}^{2}+I_{1} \lambda_{1}+I_{2}=0
$$

where

$$
I_{1}:=\frac{1}{2 \lambda_{0}} \operatorname{tr}\left(\hat{\boldsymbol{A}}_{1}+\lambda_{0} \hat{\boldsymbol{D}}_{1}\right) \quad \text { and } \quad I_{2}:=\frac{1}{4 \lambda_{0}^{2}} \operatorname{det}\left(\hat{\boldsymbol{A}}_{1}+\lambda_{0} \hat{\boldsymbol{D}}_{1}\right)
$$

are linear and quadratic invariants, respectively, which are complex numbers. From (24) two (generally distinct) roots, $\lambda_{1}^{ \pm}:=\left(-I_{1} \pm \sqrt{I_{1}^{2}-4 I_{2}}\right) / 2$, are drawn, which cause the splitting (or bifurcation) of the double semisimple eigenvalue $i \omega_{k}$ into

$$
\lambda^{ \pm}=i \omega_{k}+\varepsilon \lambda_{1}^{ \pm} .
$$


Each eigenvalue $\lambda_{1}^{ \pm}$is associated with a (distinct) eigenvector $\boldsymbol{a}^{ \pm}$; therefore $\boldsymbol{w}^{ \pm}=$ $\boldsymbol{U} \boldsymbol{a}^{ \pm}+O(\varepsilon)$, so that the indeterminacy of the eigenvectors is resolved at this order.

The undamped case. To tackle the undamped system one would most easily start from the standard form (12) of the eigenvalue problem. However, the same results can be derived from the general damped case, by letting $\hat{\boldsymbol{D}}_{1}=\mathbf{0}$ in (22). Since $\mu:=-\lambda^{2}$, or, in series form, $\mu_{0}+\varepsilon \mu_{1}+\cdots=-\left(\lambda_{0}^{2}+2 \varepsilon \lambda_{0} \lambda_{1}+\cdots\right)$, we have $\mu_{0}=-\lambda_{0}^{2}$ and $\mu_{1}:=-2 \lambda_{0} \lambda_{1}$. Therefore, (22) becomes

$$
\left(\hat{\boldsymbol{A}}_{1}-\mu_{1} \boldsymbol{I}\right) \boldsymbol{a}=\mathbf{0},
$$

which has the advantage of having real coefficients. The relevant characteristic equation,

$$
\mu_{1}^{2}-\left(\operatorname{tr} \hat{\boldsymbol{A}}_{1}\right) \mu_{1}+\operatorname{det} \hat{\boldsymbol{A}}_{1}=0,
$$

provides the roots $\mu_{1}^{ \pm}:=\left(\operatorname{tr} \hat{\boldsymbol{A}}_{1} \pm \sqrt{\operatorname{tr}^{2} \hat{\boldsymbol{A}}_{1}-4 \operatorname{det} \hat{\boldsymbol{A}}_{1}}\right) / 2$; hence

$$
\mu^{ \pm}=\omega_{k}^{2}+\varepsilon \mu_{1}^{ \pm} \text {. }
$$

Stability domains. We look for the stability domains of the trivial equilibrium in the plane of the bifurcation parameters $\alpha$ and $L$, for fixed axial load $P$ and damping $\xi$ and $\eta$, taken as auxiliary parameters. We separately address the undamped and damped cases.

Undamped system. As we said earlier, the equilibrium is stable when $\mu>0$; since, in (29), $\varepsilon \mu_{1}^{ \pm}$is a small correction of $\omega_{k}^{2}$, this happens when $\mu_{1}^{ \pm}$is real, irrespective of its sign, that is, when the discriminant of the second-degree (28) is positive. Since

$$
\operatorname{tr}^{2} \hat{\boldsymbol{A}}_{1}-4 \operatorname{det} \hat{\boldsymbol{A}}_{1}=\alpha^{2} c_{\alpha}^{2}-L^{2} c_{L}^{2},
$$

where $c_{\alpha}$ and $c_{L}$ are numerical coefficients, the stability condition reads

$$
L^{2} \leq\left(\frac{c_{\alpha}}{c_{L}}\right)^{2} \alpha^{2}
$$

This equation expresses the Nicolai paradox: when the asymmetry parameter $\alpha$ is zero (an ideal system), then an evanescent torque makes the equilibrium unstable; when, instead, a small asymmetry exists $(\alpha \neq 0)$, a small threshold $L_{c}$ exists, proportional to $\alpha$, which has to be reached by $L$ to make the system unstable. Note that the stable domain depends on the order number $k$ of the eigenvalue $\lambda_{0}=i \omega_{k}$ which bifurcates. Therefore, we have to analyze all the eigenvalues in order to determine which of them prevails in triggering instability.

Figure 1 reports the numerical results for the elliptical cross-section. Figure 1a shows the stable domain (the shaded zone) relevant to the lower eigenvalue $(k=1)$, when the axial load is zero, by comparing asymptotic (thick lines) and numerical 
(thin lines) results, the latter deriving from the exact solution of the algebraic eigenvalue problem. The domain is found to be independent of the number $N$ of trial functions used, since, due to the fact the elastic stiffness matrix is diagonal, the eigenvectors $\boldsymbol{u}_{1}$ and $\boldsymbol{u}_{2}$ are canonical vectors. The angular coefficient of the boundary lines is equal, in its absolute value, to 3.26. In the figure, a sketch on the complex plane of the four $\lambda$-eigenvalues involved (that is, the double $\pm i \omega_{1}$ eigenvalues) and their "velocities" is also given. It is seen that in the stable zone the eigenvalues are purely imaginary and distinct; on the boundary of the region they coalesce in pairs on the imaginary axis; and out of the stable zone, they separate in two pairs of stable and unstable eigenvalues. Therefore, in crossing the boundaries, a dynamic bifurcation takes place. The boundaries are a codimension- 1 geometrical locus on which the degeneracy of the eigenvalues, existing in the symmetrical unloaded system, persists. The origin of the parameter plane is therefore a codimension-2 bifurcation point.

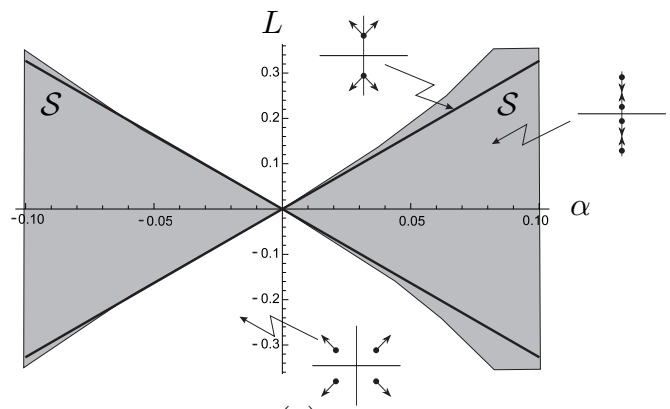

(a)

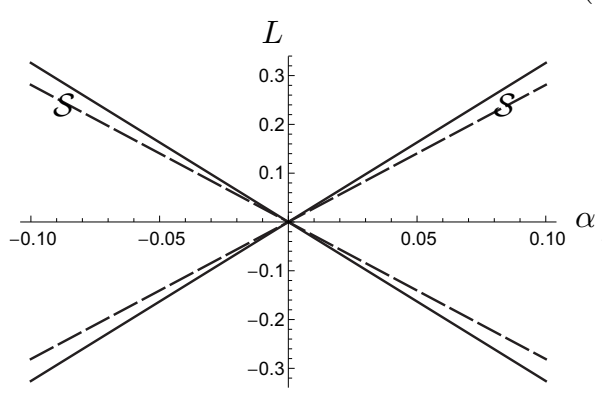

(b)

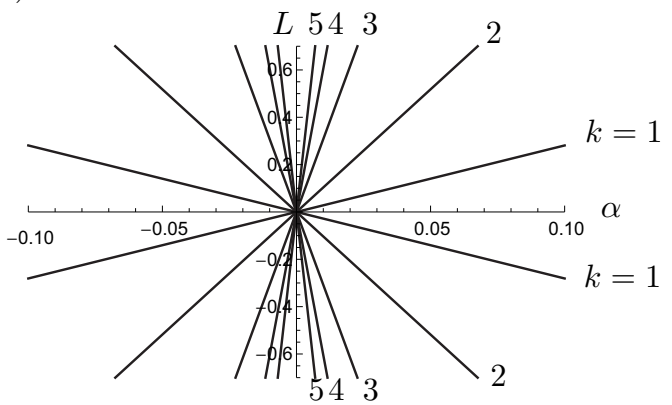

(c)

Figure 1. Stability domain $\mathscr{S}$ in the $(\alpha, L)$-plane for the undamped system subcritically loaded: (a) $k=1, P=0$ (thick line: asymptotic results; thin line: numerical results); (b) $k=1, P=0$ (solid line) and $P=P_{E} / 2$ (dashed line), with $N=5$ trial functions; (c) higher eigenvalue domains $(k=1, \ldots, 5, N=5)$ when $P=$ $P_{E} / 2$. 
Figure $1 \mathrm{~b}$ compares the domains relevant to the lower eigenvalue when $P$ is 0 and $P_{E} / 2$, showing a moderate reduction produced by the axial load (the angular coefficient reduces from 3.26 to 2.81). When $P \neq 0$, the domain depends on the discretization adopted. It was found that when $N=5$ (that is, when the system is reduced to ten degrees of freedom) a numerical convergence is reached; this number of trial functions will be therefore adopted ahead. Finally, Figure 1c compares the stability domains relevant to the higher eigenvalues $(k=1, \ldots, 5)$. It is found that the domains of higher modes include the domains of the lower modes (that is, when the lowest eigenvalue bifurcates, the higher ones are still on the imaginary axis, so that just the first mode is significant for stability).

Damped system. When the system is damped, its eigenvalues are given by (26), and the analysis, with major difficulties, must be carried out on complex quantities. The trivial equilibrium is asymptotically stable when $\operatorname{Re} \lambda^{ \pm}<0$, that is, when $\operatorname{Re} \lambda_{1}^{ \pm}<0$. In order for (24) to admit roots with real parts less than zero, the following conditions must be satisfied (the Bilharz theorem; see [Seyranian and Mailybaev 2003, p. 15]):

$$
\operatorname{Re} I_{1}>0, \quad\left(\operatorname{Im} I_{2}\right)^{2}-\operatorname{Re} I_{1} \operatorname{Im} I_{1} \operatorname{Im} I_{2}-\left(\operatorname{Re} I_{1}\right)^{2} \operatorname{Re} I_{2}<0 .
$$

Condition (32) $)_{1}$ is always satisfied when the damping coefficients are positive; $(32)_{2}$ instead gives the following (asymptotic) stability condition:

$$
L^{2}<\left(\frac{c_{\alpha}}{c_{L}}\right)^{2} \alpha^{2}+\left(\frac{c_{\xi}}{c_{L}} \xi+\frac{c_{\eta}}{c_{L}} \eta\right)^{2}
$$

where the $c$ 's are numerical coefficients. It turns out that damping, both external and internal, as well asymmetries, has a stabilizing effect on the equilibrium. If $\xi=$ $\eta=0$, then the result relevant to the undamped system is recovered. The numerical values of the $c$-coefficients are reported in Table 1 for the elliptical section and selected values of the compressive dead load $P$, when $N=5$.

Figure 2 shows the stability domain for a damped system, as compared with Figure 1 for the corresponding undamped system. As already noted in [Seyranian and Mailybaev 2011], it appears (see Figure 2a) that damping destroys the Nicolai paradox, since a nonzero torque is needed to trigger instability at $\alpha=0$. The figure also illustrates the mechanism of bifurcation. Inside the stability domain

\begin{tabular}{lccr|} 
& $c_{\alpha} / c_{L}$ & $c_{\xi} / c_{L}$ & $c_{\eta} / c_{L}$ \\
$P=0$ & 3.26 & 0.93 & 11.47 \\
$P=\frac{1}{2} P_{E}$ & 2.81 & 0.57 & 7.12 \\
\hline
\end{tabular}

Table 1. Numerical coefficients in (33) when $k=1$. 


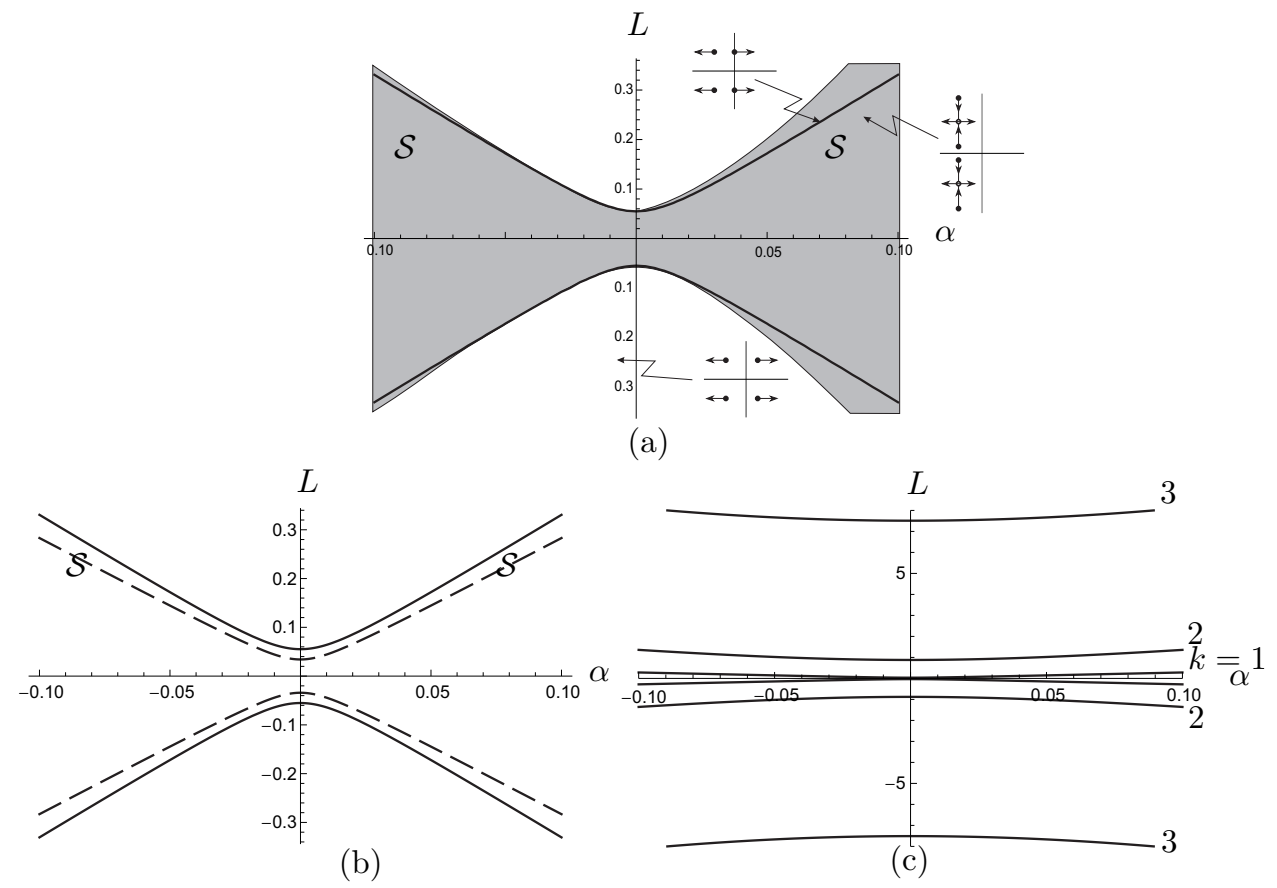

Figure 2. Stability domain $\mathscr{Y}$ in the $(\alpha, L)$-plane for the damped system $(\xi=0.01, \eta=0.004)$ : (a) $k=1, P=0$ (thick line: asymptotic results; thin line: numerical results); (b) $k=1, P=0$ (solid line) and $P=P_{E} / 2$ (dashed line), with $N=5$ trial functions; (c) higher eigenvalue domains $(k=1, \ldots, 3, N=5)$ when $P=P_{E} / 2$.

the eigenvalues are complex in the left half-plane. By approaching the boundary moving parallel to the $L$-axis, the eigenvalues approach each other by keeping their real parts constant; then, after the collision, they move in opposite directions by varying their real parts, up to crossing the imaginary axis. As for the undamped system, the effect of the axial load is weak (see Figure 2b), and the higher modes are ineffective in determining stability. However, under very particular choices of the parameters, stability can be governed by the second mode, which is consistent with results found in [Seyranian and Mailybaev 2011].

\section{Stability analysis for critically compressed beams}

We address now the stability problem of the beam when the axial load is close to the Eulerian critical load. Accordingly, since the codimension of the problem is higher, we introduce a third bifurcation parameter $\delta P:=P-P_{E}$, and look for a stability domain in the three-dimensional $(\alpha, L, \delta P)$ parameter space. 
Perturbation analysis for semisimple eigenvalues. Guided by the results obtained for the subcritically loaded beam, we introduce the rescaling

$$
(\alpha, L, \delta P, \xi, \eta) \rightarrow \varepsilon(\alpha, L, \delta P, \xi, \eta),
$$

in which all the quantities are ordered at the same level. Accordingly, the matrices $\boldsymbol{A}$ and $\boldsymbol{D}$ admit series expansions as in (15), but with new meanings for the coefficients:

$$
\begin{aligned}
\boldsymbol{A}_{0} & =\left[\begin{array}{cc}
\boldsymbol{k}_{E}+P_{E} \boldsymbol{k}_{G} & \mathbf{0} \\
\mathbf{0} & \boldsymbol{k}_{E}+P_{E} \boldsymbol{k}_{G}
\end{array}\right], \\
\boldsymbol{A}_{1} & =\boldsymbol{A}_{1_{u}}+\boldsymbol{A}_{1_{t}}, \\
\boldsymbol{A}_{1_{u}} & =\left[\begin{array}{cc}
\alpha\left(I_{y 1}-m_{1}\right) \boldsymbol{k}_{E}+\left(\delta P-\alpha m_{1} P_{E}\right) \boldsymbol{k}_{G} & L \boldsymbol{h}_{1} \\
-L \boldsymbol{h}_{1} &
\end{array}\right] \\
\boldsymbol{A}_{1_{t}} & =\left[\begin{array}{cc}
L\left(\beta_{0} \boldsymbol{h}_{1}+P_{E} \gamma_{0} \boldsymbol{h}_{2}+\gamma_{0} \boldsymbol{h}_{3}\right) & -L\left(\beta_{0} \boldsymbol{h}_{1}+P_{E} \gamma_{0} \boldsymbol{h}_{2}+\gamma_{0} \boldsymbol{h}_{3}\right) \\
\boldsymbol{D}_{1}+\left(\delta P-\alpha m_{1} P_{E}\right) \boldsymbol{k}_{G}
\end{array}\right] \\
& =\left[\begin{array}{cc}
\xi \boldsymbol{m}+\eta \boldsymbol{k}_{E} & \mathbf{0} \\
\mathbf{0} & \xi \boldsymbol{m}+\eta \boldsymbol{k}_{E}
\end{array}\right] .
\end{aligned}
$$

Here $\boldsymbol{A}_{0}$ is evaluated at the Eulerian bifurcation point, $\boldsymbol{A}_{1}$ accounts for the (firstorder) asymmetry, torque, pretwist, and axial load increment, and $\boldsymbol{D}_{1}$ describes the damping. The eigenvalue problem formally appears as in (17), with matrices updated. By assuming the series expansions (18) for the eigenvalues and eigenvectors, the perturbation (19) is still obtained. The generating problem (19) $)_{1}$, however, now admits a multiplicity-four zero eigenvalue, in addition to nonzero, purely imaginary, double eigenvalues; therefore $\lambda_{0}=0, \pm i \omega_{2}, \ldots, \pm i \omega_{N}$. Here the zero eigenvalue can be thought of as produced by the coalescence of two vanishingly small double eigenvalues $\omega_{1}= \pm i \varepsilon$, when $\varepsilon \rightarrow 0$. Since only two eigenvectors are associated with the zero eigenvalue, this latter is nonsemisimple (or defective), while the nonzero eigenvalues are semisimple. Therefore:

(1) When we take $\lambda_{0}=i \omega_{k}$, with $k=2, \ldots, N$, we recover the results of the previous section, that is, (26) for the damped case and (29) for the undamped case.

(2) When we take $\lambda_{0}=0$, the $\varepsilon$-order perturbation equation $(19)_{2}$ becomes

$$
\left(\boldsymbol{A}_{0}+\lambda_{0}^{2} \boldsymbol{I}\right) \boldsymbol{w}_{1}=-\boldsymbol{A}_{1} \boldsymbol{w}_{0},
$$

that is, it does not contain the first-order eigenvalue sensitivity $\lambda_{1}$. As a consequence, since $\boldsymbol{A}_{1} \boldsymbol{w}_{0}$ is generally out of the range of the operator, the 
compatibility condition cannot be satisfied and the equation cannot be solved! This means that the series expansion (18) lacks the ability to describe the splitting mechanism of the nonsemisimple eigenvalue, similarly to what happens for coalescent eigenvalues of more general nonconservative systems [Luongo et al. 2000; Seyranian and Mailybaev 2003].

This drawback does not manifest itself when the system is undamped, and the standard form (12) of the eigenvalue problem is used. As a matter of fact, the relevant perturbation equations read

$$
\begin{aligned}
& \varepsilon^{0}:\left(\boldsymbol{A}_{0}-\mu_{0} \boldsymbol{I}\right) \boldsymbol{w}_{0}=\mathbf{0}, \\
& \varepsilon^{1}:\left(\boldsymbol{A}_{0}-\mu_{0} \boldsymbol{I}\right) \boldsymbol{w}_{1}=-\left(\boldsymbol{A}_{1}-\mu_{1} \boldsymbol{I}\right) \boldsymbol{w}_{0} .
\end{aligned}
$$

Now, compatibility for the $\varepsilon$-order equation can be written either for $\mu_{0} \neq 0$ or $\mu_{0}=0$. The reason for this different behavior lies in the fact that $\mu_{0}=0$ is a semisimple (not defective!) root for the characteristic equation $\operatorname{det}\left(\boldsymbol{A}_{0}\right)=0$. Moreover, since $\mu=\mu_{0}+\varepsilon \mu_{1}+\cdots=-\lambda^{2}$, when $\mu_{0}=0$ then $\lambda=O\left(\varepsilon^{1 / 2}\right)$ so that a Puiseux series of the type $\lambda=\varepsilon^{1 / 2} \lambda_{1 / 2}+\cdots$ must be used, instead of a Maclaurin series. Note that, in (37), $\mu_{1}$ assumes a different meaning, according to the value of $\lambda_{0}$; it is $\mu_{1}:=-2 \lambda_{0} \lambda_{1}$ when $\lambda_{0} \neq 0$, but it is $\mu_{1}:=-\lambda_{1 / 2}^{2}$ when $\lambda_{0}=0$ !

Summarizing: in the undamped case, (29) describes the eigenvalue sensitivities, both for zero and nonzero eigenvalues; in the damped case, (26) describes the sensitivities of the nonzero eigenvalues only. To complete the analysis, we have therefore still to analyze perturbations of the quadruple zero in the damped case.

Perturbation analysis for nonsemisimple zero-eigenvalues. We tackle the problem of finding the sensitivities of the nonsemisimple quadruple zero eigenvalue by using a Puiseux series of order $\varepsilon^{1 / 2}$, that is,

$$
\lambda=\varepsilon^{1 / 2} \lambda_{1 / 2}+\varepsilon \lambda_{1}+\cdots, \quad \boldsymbol{w}=\boldsymbol{w}_{0}+\varepsilon^{1 / 2} \boldsymbol{w}_{1 / 2}+\varepsilon \boldsymbol{w}_{1}+\cdots .
$$

This problem is similar to the perturbation of a Jordan block of order 2, admitting just one eigenvector [Luongo 1993; Seyranian and Mailybaev 2003].

To ensure that the damping and bifurcation parameters appear at the same level in the perturbation scheme, we use a different ordering for them, namely

$$
(\alpha, L, \delta P) \rightarrow \varepsilon(\alpha, L, \delta P), \quad(\xi, \eta) \rightarrow \varepsilon^{1 / 2}(\xi, \eta) .
$$

Accordingly,

$$
\begin{aligned}
& \boldsymbol{A}=\boldsymbol{A}_{0}+\varepsilon \boldsymbol{A}_{1}+O\left(\varepsilon^{2}\right), \\
& \boldsymbol{D}=\varepsilon^{1 / 2} \boldsymbol{D}_{1 / 2}+O\left(\varepsilon^{3 / 2}\right),
\end{aligned}
$$


where $\boldsymbol{A}_{0}$ and $\boldsymbol{A}_{1}$ are defined in (35) 1 and (35) 2 , and $\boldsymbol{D}_{1 / 2}$ coincides with $\boldsymbol{D}_{1}$ in $(35)_{5}$.

The following perturbation equations are found:

$$
\begin{array}{lrl}
\varepsilon^{0}: & \boldsymbol{A}_{0} \boldsymbol{w}_{0}=\mathbf{0}, \\
\varepsilon^{1 / 2}: & \boldsymbol{A}_{0} \boldsymbol{w}_{1 / 2}=\mathbf{0}, \\
\varepsilon^{1}: & \boldsymbol{A}_{0} \boldsymbol{w}_{1}=-\left(\boldsymbol{A}_{1}+\lambda_{1 / 2}^{2} \boldsymbol{I}+\lambda_{1 / 2} \boldsymbol{D}_{1 / 2}\right) \boldsymbol{w}_{0} .
\end{array}
$$

The solution to the $\varepsilon^{0}$-equation is still expressed by $\boldsymbol{w}_{0}=\boldsymbol{U} \boldsymbol{a}$, in which $\boldsymbol{U}=$ $\left(\boldsymbol{u}_{1}, \boldsymbol{u}_{2}\right)$ collects the real eigenvectors associated with the zero-eigenvalue. Then, the $\varepsilon^{1 / 2}$-order equation admits a similar solution $\boldsymbol{w}_{1 / 2}=\boldsymbol{U} \boldsymbol{b}$, with $\boldsymbol{b}$ as arbitrary constants, which, however, are inessential to our (truncated) analysis. Finally, the $\varepsilon^{1}$-order equation calls for the following compatibility condition to be satisfied:

$$
\left(\hat{\boldsymbol{A}}_{1}+\lambda_{1 / 2} \hat{\boldsymbol{D}}_{1 / 2}+\lambda_{1 / 2}^{2} \boldsymbol{I}\right) \boldsymbol{a}=\mathbf{0},
$$

where

$$
\hat{A}_{1}:=U^{T} A_{1} U, \quad \hat{D}_{1 / 2}:=U^{T} D_{1 / 2} U .
$$

Like in the subcritical analysis the matrix $\hat{\boldsymbol{A}}_{1}$ does not depend on the pretwist, which therefore does not influence the stability, even close to the Eulerian load. Equation (42) is an eigenvalue problem in nonstandard form, whose characteristic equation reads

$$
\lambda_{1 / 2}^{4}+J_{1} \lambda_{1 / 2}^{3}+J_{2} \lambda_{1 / 2}^{2}+J_{3} \lambda_{1 / 2}+J_{4}=0,
$$

the invariants of which are real and assume the following expressions:

$$
\begin{array}{ll}
J_{1}:=\operatorname{tr} \hat{\boldsymbol{D}}_{1 / 2}, & J_{2}:=\operatorname{tr} \hat{\boldsymbol{A}}_{1}+\operatorname{det} \hat{\boldsymbol{D}}_{1 / 2}, \\
J_{3}:=\operatorname{det}\left(\hat{\boldsymbol{A}}_{1}+\hat{\boldsymbol{D}}_{1 / 2}\right)-\operatorname{det} \hat{\boldsymbol{A}}_{1}-\operatorname{det} \hat{\boldsymbol{D}}_{1 / 2}, & J_{4}:=\operatorname{det} \hat{\boldsymbol{A}}_{1} .
\end{array}
$$

The fourth-degree equation (44) generally admits four roots $\lambda_{1 / 2}^{(i)}, i=1, \ldots, 4$. They describe the bifurcation of the quadruple zero-eigenvalue in four distinct roots $\lambda^{(i)}=\varepsilon^{1 / 2} \lambda_{1 / 2}^{(i)}+O(\varepsilon)$. To each of them, a distinct eigenvector $\boldsymbol{a}^{(i)}$ is associated, so that four distinct eigenvectors $\boldsymbol{w}^{(i)}=\boldsymbol{U} \boldsymbol{a}^{(i)}+O\left(\varepsilon^{1 / 2}\right)$ are found.

When damping vanishes $\left(\hat{\boldsymbol{D}}_{1 / 2}=\mathbf{0}\right)$, then $J_{1}=J_{3}=0$, and (44) reduces to

$$
\lambda_{1 / 2}^{4}+\left(\operatorname{tr} \hat{\boldsymbol{A}}_{1}\right) \lambda_{1 / 2}^{2}+\operatorname{det} \hat{\boldsymbol{A}}_{1}=0
$$

which is identical to (28), once $\mu_{1}=-\lambda_{1 / 2}^{2}$ is accounted for, as discussed before. Therefore, the present algorithm, tailored to damped systems, also works for undamped systems. 
Stability domains. In evaluating the stability domains, we have to distinguish bifurcations of the lower zero-eigenvalue, governed by (28) or (44) (holding in the undamped and damped cases, respectively), and bifurcations of the higher nonzero eigenvalues, governed by (28) or (24). We will focus our attention on the zeroeigenvalue, and then check the behavior of higher eigenvalues.

Undamped system. Stability requires that $\mu$ be real and positive. Since $\mu=\mu_{0}+$ $\varepsilon \mu_{1}^{ \pm}+\cdots$, when $\mu_{0}=0, \mu_{1}^{ \pm}$itself must be real and positive (unlike the $P<P_{E}$ case, in which it only was required to be real). This occurrence is satisfied when

$$
\operatorname{tr}^{2} \hat{\boldsymbol{A}}_{1}-4 \operatorname{det} \hat{\boldsymbol{A}}_{1}>0, \quad \operatorname{tr} \hat{\boldsymbol{A}}_{1}>0, \quad \operatorname{det} \hat{\boldsymbol{A}}_{1}>0 \text {. }
$$

When these inequalities are written out in terms of bifurcation parameters, they assume the form

$$
\begin{aligned}
c_{\alpha} \alpha^{2}+c_{L} L^{2} & >0, \\
b_{\alpha} \alpha+b_{\delta} \delta P & >0, \\
d_{L} L^{2}+d_{\alpha} \alpha^{2}+d_{\alpha \delta} \alpha \delta P+d_{\delta} \delta P^{2} & >0,
\end{aligned}
$$

where the $b, c$, and $d$ are numerical coefficients. For the elliptical cross-section, with $N=5$, these assume the values shown in Table 2 . When the inequalities are replaced by equalities, we obtain the equations of, in order: a pair of planes parallel to the $\delta P$-axis (and containing the origin), a plane parallel to the $L$-axis (ditto); and a cone (with vertex at the origin). The four surfaces bound the threedimensional domain shown in Figure 3 from different views, where contour plots $P=$ const. are drawn to facilitate the reading of the image. The equilibrium is stable in the volume subtended by the portion of conical surface represented in the picture. This figure also contains a sketch of the four nearly zero $\lambda$-eigenvalues involved in the bifurcation, represented on an $\alpha=$ const. plane. On the vertical planes two complex conjugate eigenvalues cross the imaginary axes, so that a dynamic bifurcation occurs; on the cone surface, a zero eigenvalue crosses the axis, so that a static bifurcation takes place; at the two straight lines, intersections of the vertical planes and the cone, the four eigenvalues are coincident at the origin, so that the lines select a codimension- 2 family of degenerate systems around which static and dynamic bifurcations are expected to exist. The origin of parameter space is therefore a codimension-3 bifurcation point. The figure also illustrates the mechanisms of bifurcations along three different paths. Along path I ( $L$ increasing),

$\begin{array}{cccccccc}c_{\alpha} & c_{L} & b_{\alpha} & b_{\delta} & d_{L} & d_{\alpha} & d_{\alpha \delta} & d_{\delta} \\ 6.24 & -1 & 4.94 & -1 & 0.053 & 1 & -0.54 & 0.055\end{array}$

Table 2. Numerical coefficients in (48). 


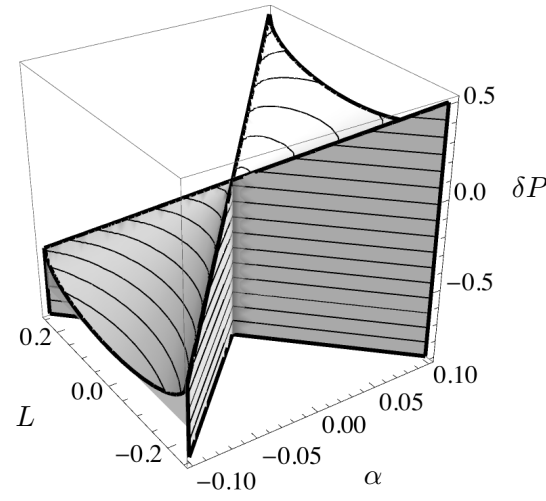

(a)

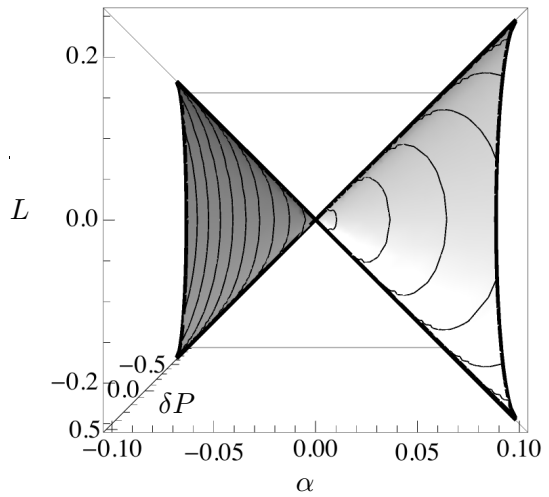

(c)

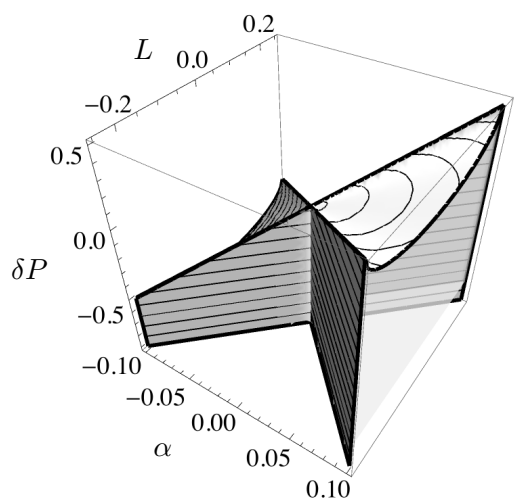

(b)

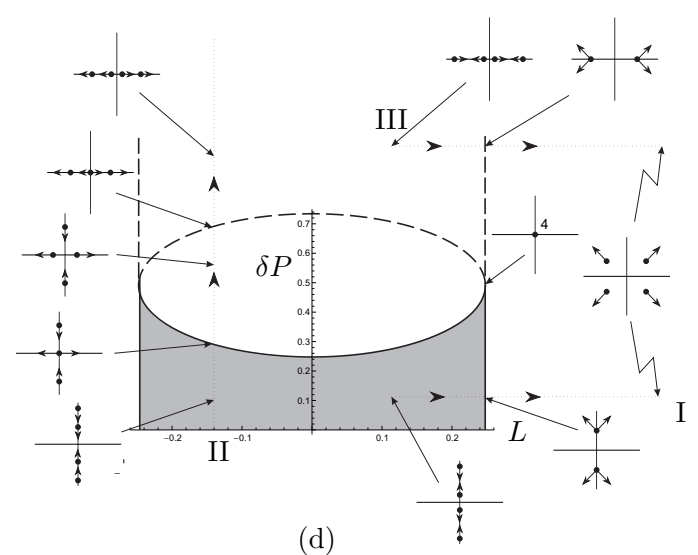

(d)

Figure 3. Stability domain in $(\alpha, L, \delta P)$-space for the undamped system critically loaded: (a)-(c) different views, and (d) sketches of the four nearly zero eigenvalues on the $\alpha=0.1$ plane.

the eigenvalue behavior is identical to that previously illustrated for the subcritical regime. Along path II ( $\delta P$ increasing), when the conical surface is crossed, two zero eigenvalues occur, splitting in opposite real eigenvalues, while the remaining two eigenvalues are purely imaginary; a successive static bifurcation occurs at the upper branch. Path III illustrates how the velocities of the quadruple zero eigenvalue depend on the region entered by the variation of the parameters.

As a general comment on the effect of the axial load on stability, we observe that, when $\alpha>0$ (that is, when the beam is stiffened by asymmetries), overcritical states $P>P_{E}$ can be visited, except for $L=0$, for which the static bifurcation always occurs at $P=P_{E}$ in the plane of minimum stiffness. When $\alpha<0$ (that is, when the beam is weakened by asymmetries) instead, the static bifurcation occurs at a subcritical value $P>P_{E}$. As a main result, the axial load is ineffective 
on the critical torque, which only depends on the asymmetry $\alpha$, thus confirming the weak dependence we found in the subcritical field (recall Figure 1b). The reverse, however, is not true! Indeed, for a given $\alpha$, a moderately small torque has a beneficial effect on the stability, since it increases the maximum axial load bearable by the beam.

When higher eigenvalues were studied, it was found that the relevant stability domains include that relevant to the first zero-eigenvalue. Therefore stability is only governed by this latter.

Damped system. The damped system is asymptotically stable when its eigenvalues have negative real parts. Since the zero eigenvalue bifurcates into four eigenvalues $\lambda=\varepsilon^{1 / 2} \lambda_{1 / 2}^{(i)}+\cdots$, we have to require that all of them move to the left part of the complex plane. This is ensured by the conditions stated by the Routh-Hurwitz criterion, when applied to the real-coefficient fourth-degree equation (44), namely

$$
\begin{gathered}
J_{i}>0, \quad i=1, \ldots, 4, \\
J_{1} J_{2}-J_{3}>0, \quad J_{1} J_{2} J_{3}-J_{3}^{2}-J_{1}^{2} J_{4}>0 .
\end{gathered}
$$

Since the damping coefficients are positive, only three out of six conditions are meaningful, and they turn out to be of the following form:

$$
\begin{aligned}
c_{\alpha} \alpha+c_{\delta} \delta P & >0, \\
b_{L} L^{2}+b_{\alpha} \alpha^{2}+b_{\alpha \delta} \alpha \delta P+b_{\delta} \delta P^{2} & >0, \\
d_{L} L^{2}+d_{\alpha} \alpha\left(d_{\xi} \xi+d_{\eta} \eta\right)^{2}+d_{\alpha \alpha} \alpha^{2}+d_{\delta} \delta P\left(d_{\xi} \xi+d_{\eta} \eta\right)^{2} & >0,
\end{aligned}
$$

where the $b, c$, and $d$ 's are numerical coefficients. Their values are reported in Table 3 for an elliptical cross-section and $N=5$. The relevant stability domain is shown in Figure 4 from different views. By comparing these with Figure 3, it is seen that damping has a smoothing effect close to the vertical axis, as already noticed in the subcritical regime (see Figure 2); moreover, the cone is unaffected by damping. Figure 4 also shows the eigenvalues at the boundaries of the domain, and the different mechanisms of bifurcation. Dynamic and static bifurcations still occur at the lateral surface of the domain and at the cone, respectively, as for the undamped case; however, the coalescence on the imaginary axis, peculiar to circulatory systems, is now destroyed. Similarly, the two intersection lines for the origin are loci of double (no longer quadruple) zero eigenvalues. Analysis of higher eigenvalues confirms that they are not relevant in determining stability.

\begin{tabular}{|cccccccccccc|}
\hline$c_{\alpha}$ & $c_{\delta}$ & $b_{L}$ & $b_{\alpha}$ & $b_{\alpha \delta}$ & $b_{\delta}$ & $d_{L}$ & $d_{\alpha}$ & $d_{\alpha \alpha}$ & $d_{\delta}$ & $d_{\xi}$ & $d_{\eta}$ \\
4.94 & -1 & 0.053 & 1 & -0.54 & 0.055 & -1 & 0.93 & 6.24 & -0.19 & 1 & 13.42 \\
\hline
\end{tabular}

Table 3. Numerical coefficients in (50). 


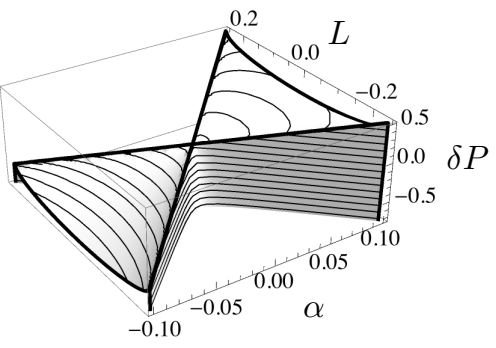

(a)

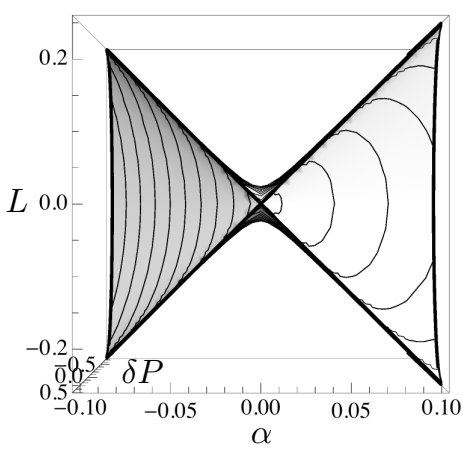

(c)

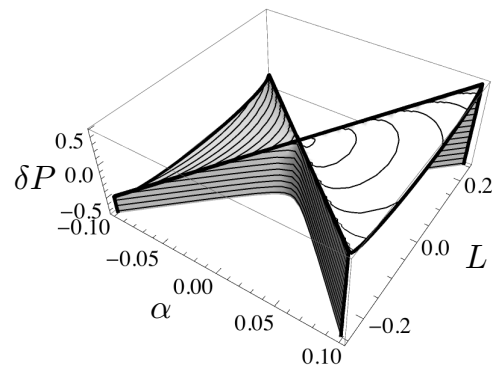

(b)

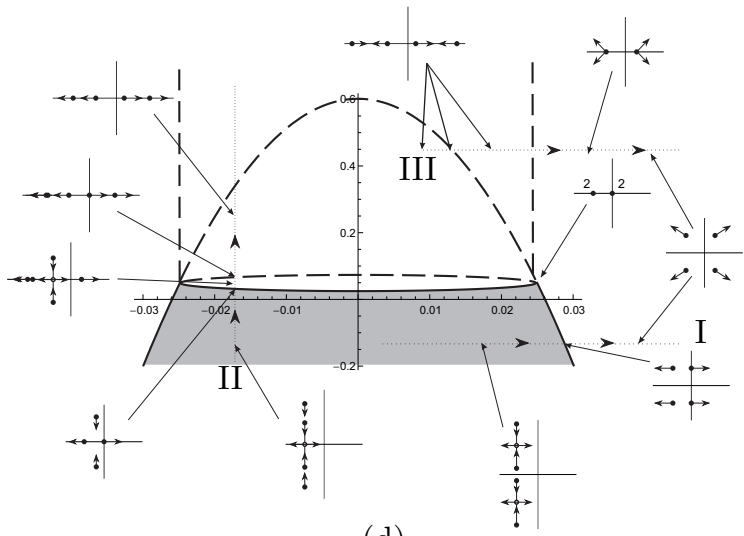

(d)

Figure 4. Stability domain in $(\alpha, L, \delta P)$-space for a damped system critically loaded ( $\xi=0.01, \eta=0.005)$ : (a) - (c) different views, and (d) sketches of the four nearly zero eigenvalues on the $\alpha=0.01$ plane.

Comparison between asymptotic and numerical results for the algebraic eigenvalue problem is performed in Figure 5 for some $L=$ const. cross-sections of the three-dimensional solid domains in Figures 3 and 4. Excellent agreement is found in the regions considered.

\section{Conclusions}

The Nicolai problem of the stability of a quasisymmetric cantilever beam in three dimensions, loaded by a compressive dead load and a follower torque, has been considered. Attention has been focused on the effects of damping, of both external and internal types, and on the axial load, both lower than or close to the Eulerian critical value. The problem has first been formulated in a strong form, by accounting for the pretwist produced by the torque when the beam is rectilinear, and then recast in a weak form via the Galerkin method, by using the planar eigenmodes of the stress-free beam as trial functions. 


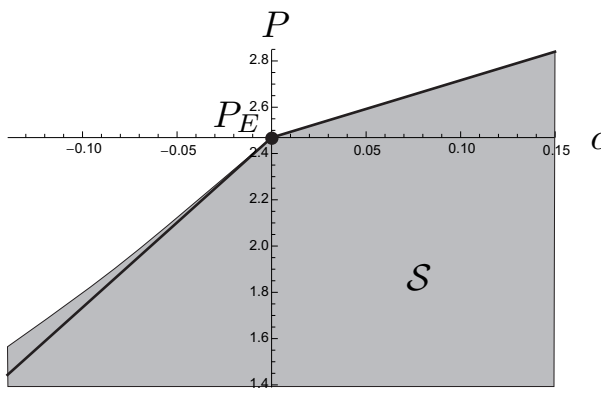

(a)

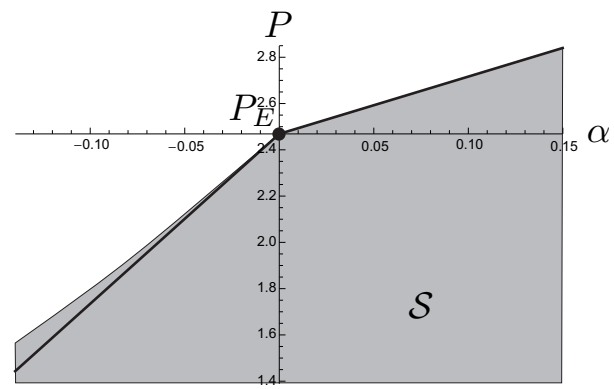

(c)

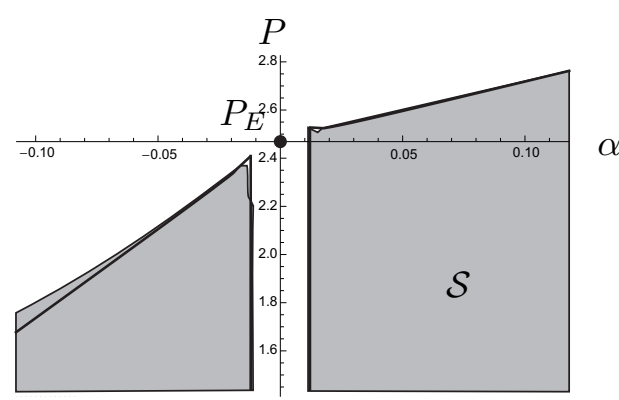

(b)

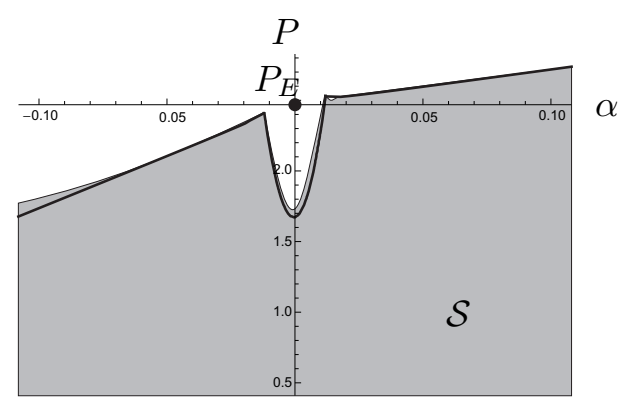

(d)

Figure 5. Comparison between asymptotic (thick lines) and numerical (thin lines) stability regions for a critically loaded system:

(a) undamped system, $L=0$; (b) undamped system, $L=0.03$;

(c) damped system $(\xi=0.01, \eta=0.005), L=0$; (d) damped system $(\xi=0.01, \eta=0.005), L=0.03$.

The eigenvalue problem, governing the motion around the rectilinear configuration, has been derived, both in nonstandard form (for damped systems) and in standard form (for undamped systems). Perturbation methods have been used to solve it, and are able to analytically describe the dependence of the eigenvalues on the bifurcation parameters. An ideally undamped symmetric system, consisting of a beam with equal geometrical characteristics in the two principal planes of inertia, axially loaded but free of torque, has been considered as the generator system for the perturbation process. The unperturbed eigenvalues, due to the symmetry, are double semisimple purely imaginary eigenvalues associated with two independent eigenvectors, each describing an oscillation mode in a different inertial plane. An exception, however occurs at the Eulerian load, where two semisimple eigenvalues coalesce at zero, thus giving rise to a quadruple non-semisimple root, at which only two eigenvectors are associated (the buckling modes in the two planes). 
Accordingly, while semisimple eigenvalues can be tackled by standard perturbation methods, entailing the use of Taylor series, the nonsemisimple zero eigenvalue calls for the use of Puiseux series. In undamped systems, however, the overdegeneracy of the quadruple eigenvalue does not entail any drawbacks, if the eigenvalue problem is stated in standard form, since here it still appears as semisimple.

Stability domains have been built up both for subcritically loaded beams, in the two-dimensional (asymmetry and torque) parameter plane, and for critically loaded beams in the three-dimensional (asymmetry-torque-load) parameter space. The following main results have been drawn.

The stability domains are governed mostly by bifurcations of the lowest eigenvalue of the ideal system, purely imaginary or zero. Therefore, although higher eigenvalues can bifurcate into unstable eigenvalues, this happens in regions of parameters which are already unstable, due to bifurcation of the lowest eigenvalue. However, special systems have been detected in a narrow region of the damping parameters plane in which the second mode is leading.

In the subcritical range, the stability domain of the undamped system is a portion of the plane bounded by two straight lines passing through the origin and including the asymmetry axis. Therefore, if the system is symmetric, an evanescent torque causes instability (the Nicolai paradox); however, finite small asymmetries entail a finite (but small) threshold of the torque, which increases with the asymmetry. The straight lines are loci of systems admitting a double semisimple eigenvalue. When they are crossed from the inside, a dynamic bifurcation takes place. When damping is added, the lines change in smooth curves external to the origin, so that a finite critical torque exists even for symmetric systems, that is, damping has a stabilizing effect, which destroys the paradox of Nicolai. The effect of the axial load on the torque is weak.

In the critical range, the stability domain is a portion of the space which is bounded by three planes and a conical surface. Both static and dynamic bifurcations can occur through these surfaces. Axial loads have no effect on the critical torque, which only depends on the asymmetry magnitude. In contrast, a small torque has a beneficial effect on the static instability triggered by the axial force. When damping is introduced, the sharp edges of the stability domain are smoothed, and a stabilizing effect on the dynamic instability is produced.

In a remarkable result, the stability of the Nicolai beam has been found not to depend on the pretwist, to a first-order approximation, neither in the subcritical nor in the critical range.

Asymptotic analysis gives results in excellent agreement with numerical solutions of the algebraic eigenvalue problem relevant to the discretized beam. 


\section{Appendix A: Geometrical characteristics of perturbed cross-sections}

Let us consider a cross-section having identical elastogeometrical characteristics with respect to the principal axes $x$ and $y$. When one of the two major dimensions is perturbed by a small nondimensional parameter $\|\alpha\| \ll 1$, its characteristics modify into

$$
\begin{aligned}
A & =A_{0}+\alpha A_{1}, & I_{x} & =I_{0}+\alpha I_{x_{1}}, \\
I_{y} & =I_{0}+\alpha I_{y_{1}}+O\left(\alpha^{2}\right), & J & =J_{0}+\alpha J_{1}+O\left(\alpha^{2}\right) .
\end{aligned}
$$

Since the mass per unit length is proportional to the area, it is

$$
m=m_{0}+\alpha m_{1}
$$

where $m_{0}=m_{1}=A_{0} \varrho, \varrho$ being the density. The magnitudes $m_{0}$ and $I_{0}$ have been used in (3) to define nondimensional quantities. Two examples are given here.

Elliptical cross-section. A slightly eccentric ellipse is considered, with half-axes $a=R(1+\alpha)$ and $b=R$, along $x$ and $y$, respectively. When $\alpha=0$, the cross-section becomes circular with radius $R$. The (dimensional) geometric characteristics read as in (A.1), where

$$
\begin{array}{lll}
A_{0}:=\pi R^{2}, & I_{0}:=\frac{\pi R^{4}}{4}, & J_{0}:=\frac{\pi R^{4}}{2}, \\
A_{1}:=\pi R^{2}, & I_{x_{1}}:=\frac{\pi R^{4}}{4}, & I_{y_{1}}:=\frac{3 \pi R^{4}}{4},
\end{array}
$$

Consequently, the nondimensional geometrical characteristics and mass per unit length reads

$$
\begin{aligned}
& \tilde{I}_{x}=1+\alpha, \quad \tilde{I}_{y}=1+3 \alpha+O\left(\alpha^{2}\right), \\
& \beta=\beta_{0}+O\left(\alpha^{2}\right), \quad \gamma=\gamma_{0}(1-2 \alpha)+O\left(\alpha^{2}\right), \quad \tilde{m}=1+\alpha,
\end{aligned}
$$

where $\beta_{0}:=2 E / G$ and $\gamma_{0}:=E / G$.

Rectangular cross-section. We consider a rectangle of sides $a=h(1+\alpha)$ and $b=h$, along $x$ and $y$, respectively. When $\alpha=0$, the cross-section becomes a square, of side $h$. The (dimensional) geometric characteristics are given by (A.1), where

$$
\begin{array}{lll}
A_{0}:=h^{2}, & I_{0}:=\frac{h^{4}}{12}, & J_{0}:=0.141 h^{4}, \\
A_{1}:=h^{2}, & I_{x_{1}}:=\frac{h^{4}}{12}, & I_{y_{1}}:=\frac{h^{4}}{4},
\end{array}
$$


and the corresponding nondimensional quantities become

$$
\begin{aligned}
& \tilde{I}_{x}=1+\alpha, \quad \tilde{I}_{y}=1+3 \alpha+O\left(\alpha^{2}\right), \\
& \beta=\beta_{0}+O(\alpha), \quad \gamma=\gamma_{0}+O(\alpha), \quad \tilde{m}=1+\alpha,
\end{aligned}
$$

where $\beta_{0}:=2.364 E / G$ and $\gamma_{0}:=1.182 E / G$.

\section{Appendix B: Trial functions used in the Galerkin projection}

The trial functions used in (6) are taken as the modes of the free oscillations of a planar unprestressed cantilever. The relevant boundary value problem is

$$
\phi^{I V}-\omega^{2} \phi=0, \quad \phi(0)=\phi^{\prime}(0)=\phi^{\prime \prime}(1)=\phi^{\prime \prime \prime}(1)=0,
$$

where $\omega$ is a nondimensional natural frequency. The solution reads

$$
\phi(z)=c\left\{\sin (\gamma z)-\sinh (\gamma z)-\frac{[\sin (\gamma)+\sinh (\gamma)][\cos (\gamma z)-\cosh (\gamma z)]}{\cos (\gamma)+\cosh (\gamma)}\right\}
$$

where $\gamma:=\sqrt{\omega}$ is a root of the characteristic equation

$$
\cos (\gamma) \cosh (\gamma)+1=0
$$

Moreover $c$ is an arbitrary constant, to be determined via the normalization condition

$$
\int_{0}^{1} \phi^{2}(z) d z=1
$$

Table 4 reports the values of $\gamma$ and $c$ for the first five modes. The eigenfunctions satisfy the orthogonality conditions:

$$
\int_{0}^{1} \phi_{i} \phi_{j} d z=\delta_{i j}, \quad \int_{0}^{1} \phi_{i}^{\prime \prime} \phi_{j}^{\prime \prime} d z=\delta_{i j} \omega_{i}^{2},
$$

where $\delta_{i j}$ is the Kronecker symbol.

\begin{tabular}{|cccccc|}
\hline & \multicolumn{1}{c}{1} & \multicolumn{1}{c}{2} & \multicolumn{1}{c}{3} & \multicolumn{1}{c}{4} & 5 \\
$\gamma$ & 1.8751 & 4.6941 & 7.8548 & 10.9955 & 14.1372 \\
$c$ & 0.734098 & 1.018460 & 0.999220 & 1.000040 & 0.999996 \\
\hline
\end{tabular}

Table 4. Values of $\gamma$ and $c$ in (B.2) for the first five modes. 


\section{Appendix C: Equations of motion}

The motion of the beam, taking into account inertial effects, is governed by the following equations, derived in [Bolotin 1963]:

$$
\begin{aligned}
T_{x}^{\prime}-\tilde{\kappa}_{z} T_{y}-\kappa_{y} P+f_{x} & =0, \\
T_{y}^{\prime}+\tilde{\kappa}_{z} T_{x}+\kappa_{x} P+f_{y} & =0, \\
M_{x}^{\prime}-\tilde{\kappa}_{z} M_{y}+\kappa_{y} L-T_{y} & =0, \\
M_{y}^{\prime}+\tilde{\kappa}_{z} M_{x}-\kappa_{x} L+T_{x} & =0,
\end{aligned}
$$

where $T_{x}$ and $T_{y}$ are the shear forces, $M_{x}$ and $M_{y}$ are the bending moments, $f_{x}=$ $-m \ddot{u}$ and $f_{y}=-m \ddot{v}$ are the inertial forces, $\kappa_{x}$ and $\kappa_{y}$ are the curvatures of the beam in the two principal inertial planes, and $\tilde{\kappa}_{z}=L /(G J)$ is the pretwisting angle. All other symbols and notations appearing in the previous and subsequent equations are the same as those specified in Section 1.

The kinematic relations are [Bolotin 1963]

$$
\varphi=-v^{\prime}-\tilde{\kappa}_{z} u, \quad \psi=u^{\prime}-\tilde{\kappa}_{z} v, \quad \kappa_{x}=\varphi^{\prime}-\tilde{\kappa}_{z} \psi, \quad \kappa_{y}=\psi^{\prime}+\tilde{\kappa}_{z} \varphi,
$$

where $\varphi$ and $\psi$ are the rotations. From these, the curvatures are expressed in terms of displacements:

$$
\begin{aligned}
& \kappa_{x}=-v^{\prime \prime}-2 \tilde{\kappa}_{z} u^{\prime}+\tilde{\kappa}_{z}^{2} v, \\
& \kappa_{y}=u^{\prime \prime}-2 \tilde{\kappa}_{z} v^{\prime}-\tilde{\kappa}_{z}^{2} u .
\end{aligned}
$$

To account for internal damping, the longitudinal unit strain is written as $\varepsilon=$ $\kappa_{x} y-\kappa_{y} x$. By using the Kelvin-Voigt constitutive law, that is, $\sigma=E \varepsilon+\eta \dot{\varepsilon}$, where $\sigma$ is the normal stress, $\varepsilon$ the longitudinal unit strain, and $\eta$ the viscosity coefficient, and integrating over the area $A$ of the cross-section, we find

$$
\begin{aligned}
& M_{x}=\int_{A} \sigma y d A=E I_{x} \kappa_{x}+\eta I_{x} \dot{\kappa}_{x}, \\
& M_{y}=-\int_{A} \sigma x d A=E I_{y} \kappa_{y}+\eta I_{y} \dot{\kappa}_{y} .
\end{aligned}
$$

Then, differentiating the last two equations of (C.1) with respect to the space variable and using (C.4) and (C.1) (solved with respect to $T_{x}^{\prime}, T_{y}^{\prime}, T_{x}$, and $T_{y}$ ), we obtain

$$
\begin{aligned}
& E I_{x} \kappa_{x}^{\prime \prime}+\left(L-2 \tilde{\kappa}_{z} E I_{y}\right) \kappa_{y}^{\prime}+\left.P-\tilde{\kappa}_{z}^{2} E I_{x}+\tilde{\kappa}_{z} L\right) \kappa_{x} \\
&+\eta E I_{x} \dot{\kappa}_{x}^{\prime \prime}-2 \tilde{\kappa}_{z} \eta I_{y} \dot{\kappa}_{y}^{\prime}-\tilde{\kappa}_{z}^{2} \eta I_{x} \dot{\kappa}_{x}+f_{y}=0, \\
& E I_{y} \kappa_{y}^{\prime \prime}-\left(L-2 \tilde{\kappa}_{z} E I_{x}\right) \kappa_{x}^{\prime}+(\left.P-\tilde{\kappa}_{z}^{2} E I_{y}+\tilde{\kappa}_{z} L\right) \kappa_{y} \\
&+\eta E I_{x} \dot{\kappa}_{y}^{\prime \prime}+2 \tilde{\kappa}_{z} \eta I_{x} \dot{\kappa}_{x}^{\prime}-\tilde{\kappa}_{z}^{2} \eta I_{y} \dot{\kappa}_{y}-f_{x}=0 .
\end{aligned}
$$


These equations are further simplified by assuming (see [Seyranian et al. 2014]) that the displacements, curvatures, pretwisting $\tilde{\kappa}_{z}$, torque, and viscosity coefficient are small quantities of the first order, while third- and higher-order terms are neglected. By adding an external damping $\xi$, which accounts for the interaction of the beam with the surrounding air, the field equations (1) follow.

The boundary conditions at the clamped end of the beam require that the displacements $u$ and $v$ and rotations $\varphi$ and $\psi$ vanish (by $(2)_{2}$ ). The boundary conditions at the free end require that the bending moments vanish:

$$
M_{x}=0, \quad M_{y}=0,
$$

and that the shear forces equate the projection of the gravitational force onto the principal inertial axes in the current configuration:

$$
T_{x}=P \psi, \quad T_{y}=-P \varphi .
$$

By using (C.4) and (C.3) in (C.6) and linearizing, $(2)_{2}$ are obtained; then, by using $(\mathrm{C} .1)_{3},(\mathrm{C} .1)_{4},(\mathrm{C} .3),(\mathrm{C} .4)$, and (C.6) and linearizing, $(2)_{3}$ follow.

\section{Appendix D: Independence of stability of the pretwist}

We prove that, to the first asymptotic order, the stability of the Nicolai beam does not depend on the pretwist. To this end, it will be sufficient to prove that the contribution of the pretwist to the matrix $\hat{\boldsymbol{A}}_{1}$ that appears in (23) and (43) vanishes.

Remembering that $\boldsymbol{A}_{1}=\boldsymbol{A}_{1_{u}}+\boldsymbol{A}_{1_{t}}$, (see (16) 2 and (35) $)$, we obtain

$$
\hat{\boldsymbol{A}_{1}}=\boldsymbol{U}^{T} \boldsymbol{A}_{1_{u}} \boldsymbol{U}+\boldsymbol{U}^{T} \boldsymbol{A}_{1_{t}} \boldsymbol{U} .
$$

Since, moreover,

$$
U=\left[\begin{array}{cc}
w & 0 \\
0 & w
\end{array}\right]
$$

the contribution of the pretwist to $\hat{A}_{1}$ is

$$
\begin{aligned}
& \boldsymbol{U}^{T} \boldsymbol{A}_{1_{t}} \boldsymbol{U} \\
& =L\left[\begin{array}{cc}
\mathbf{0} & \boldsymbol{w}^{T}\left(\left(\beta_{0} \boldsymbol{h}_{1}+P_{E} \gamma_{0} \boldsymbol{h}_{2}+\gamma_{0} \boldsymbol{h}_{3}\right)\right) \boldsymbol{w} \\
-\boldsymbol{w}^{T}\left(\left(\beta_{0} \boldsymbol{h}_{1}+P_{E} \gamma_{0} \boldsymbol{h}_{2}+\gamma_{0} \boldsymbol{h}_{3}\right)\right) \boldsymbol{w} & \mathbf{0}
\end{array}\right] .
\end{aligned}
$$

By using definition $(10)_{5}$ for the $\boldsymbol{h}_{2}$ matrix, integrating by parts, and using the geometric boundary conditions, it is easy to check that $h_{2_{i j}}=-h_{2_{j i}}$, that is, that $\boldsymbol{h}_{2}$ is antisymmetric. Analogously, by considering the matrix $\beta_{0} \boldsymbol{h}_{1}+\gamma_{0} \boldsymbol{h}_{3}$, with $\boldsymbol{h}_{1}$ and $\boldsymbol{h}_{3}$ given by $(10)_{4}$ and $(10)_{6}$, accounting for $\beta_{0}=2 \gamma_{0}$, integrating by parts and using the mechanical boundary conditions satisfied by the trial functions adopted, it follows that $2 h_{1_{i j}}+h_{3_{i j}}=-2 h_{1_{j i}}-h_{3_{j i}}$, that is, $\beta_{0} \boldsymbol{h}_{1}+\gamma_{0} \boldsymbol{h}_{3}$ is antisymmetric. Therefore, the off-diagonal blocks in (D.1), and therefore the matrix itself, vanish. 


\section{Acknowledgments}

This work was supported by a grant from the Italian Ministry of Education, Universities and Research, under the PRIN10-11 program, project N. 2010MBJK5B. Seyranian expresses his gratitude for the scientific visit to University of L'Aquila in June and July of 2012, supported by Regional Funds PO FSE Abruzzo 2007-2013.

\section{References}

[Bolotin 1963] V. V. Bolotin, Nonconservative problems of the theory of elastic stability, Pergamon, New York, 1963.

[Leipholz 1974] H. H. E. Leipholz, “On the convergence of Ritz and Galerkin's method in the case of certain nonconservative systems and using admissible coordinate functions", Acta Mech. 19:1-2 (1974), 57-76.

[Luongo 1993] A. Luongo, "Eigensolutions sensitivity for nonsymmetric matrices with repeated eigenvalues", AIAA J. 31:7 (1993), 1321-1328.

[Luongo et al. 2000] A. Luongo, A. Paolone, and A. Di Egidio, "Sensitivities and linear stability analysis around a double-zero eigenvalue”, AIAA J. 38:4 (2000), 702-710.

[Nicolai 1928] E. L. Nicolai, “Об устойчивости прямолинейной формы равновесия сжатого и скрупенного стержня (On the stability of the rectilinear equilibrium shape of a rod under compression and torsion)", Izvestiia Leningradskogo Politekhnicheskogo Instituta Imeni M. I. Kalinina 31 (1928), 201-231. Reprinted as pp. 356-387 in his Труды по механике (Mechanical works), Gostekhizdat, Moscow, 1955.

[Nicolai 1929] E. L. Nicolai, "K вопросу об устойчивости скрупенного стержня (On the stability problem for a rod under torsion)", Vestnik Mekhaniki i Prikladnoi Matematiki 1 (1929), 41-58. Reprinted as pp. 388-406 in his Труды по механике (Mechanical works), Gostekhizdat, Moscow, 1955.

[Seyranian and Mailybaev 2003] A. P. Seyranian and A. A. Mailybaev, Multiparameter stability theory with mechanical applications, Series on Stability, Vibration and Control of Systems, Series A 13, World Scientific, River Edge, NJ, 2003.

[Seyranian and Mailybaev 2011] A. P. Seyranian and A. A. Mailybaev, "Paradox of Nicolai and related effects", Z. Angew. Math. Phys. 62:3 (2011), 539-548.

[Seyranian et al. 2014] A. P. Seyranian, A. Di Egidio, A. Contento, and A. Luongo, "Solution to the problem of Nicolai", J. Sound Vib. 333:7 (2014), 1932-1944.

[Zienkiewicz et al. 2005] O. C. Zienkiewicz, R. L. Taylor, and J. Z. Zhu, The finite element method: its basis and fundamentals, 6th ed., Butterworth-Heinemann, Oxford, 2005.

Received 7 Mar 2013. Revised 13 Nov 2013. Accepted 31 Dec 2013.

ANGELO LUONGO: angelo. luongo@univaq.it

Dipartimento di Ingegneria Civile, Edile-Architettura e Ambientale, Università degli studi dell'Aquila, Via Giovanni Gronchi 18, Zona industriale di Pile, 67100 L'Aquila, Italy

MANUEL FERRETTI: manuel.ferretti@univaq.it

Dipartimento di Ingegneria Civile, Edile-Architettura e Ambientale, Università degli studi dell'Aquila, Via Giovanni Gronchi 18, Zona industriale di Pile, 67100 L'Aquila, Italy

ALEXANDER P. SEYRANIAN: aseyranian@mail.ru

Institute of Mechanics, Moscow State Lomonosov University, Michurinsky pr. 1, Moscow, 119192, Russia 


\section{EDITORIAL BOARD}

ANTONIO CARCATERRA ERIC A. CARLEN

FRANCESCO DELL'ISOLA

RAFFAELE ESPOSITO

ALBERT FANNJIANG

Gilles A. FrancFort

PiERANGElo MARCATI

JEAN-JACQUES MARIGO

PETER A. MARKOWICH MARTIN OSTOJA-STARZEWSKI

PIERRE SEPPECHER

DAVID J. STEIGMANN

PAUl STEINMANN

PierRe M. Suquet

\section{MANAGING EDITORS}

MICOL AMAR

CORRADO LATTANZIO

ANGELA MADEO

MARTIN OSTOJA-STARZEWSKI

\section{ADVISORY BOARD}

ADNAN AKAY

Holm AltenbaCH

MICOL AMAR

HARM ASKES

TEODOR ATANACKOVIĆ

VICTOR BERDICHEVSKY

GuY BouchitTÉ

ANDREA BRAIDES

ROBERTO CAMASSA

MAURO CARFORE

ERIC DARVE

FELIX DARVE

ANNA DE MASI

Gianpietro Del Piero

EMMANUELE Di BENEDETTO

BERNOLD FIEDLER

IRENE M. GAMBA

SERGEY GAVRILYUK

TIMOTHY J. HEALEY

DOMINIQUE JEULIN

ROGER E. KHAYAT

CORRADO LATTANZIO

ROBERT P. LIPTON

ANGELO LUONGO

ANGEla MadeO

JUAN J. MANFREDI

CARLO MARCHIORO

GÉrard A. MAUGin

ROBERTO NATALINI

PATRIZIO NEFF

ANDREY PIATNITSKI

ERrico Presutti

MARIO PUlVIRENTI

LuCiO RuSSO

Miguel A. F. SANJUAN

Patrick SElvadurai

ALEXANDER P. SEYRANIAN

MIROSLAV ŠILHAVÝ

GUIDO SWEERS

ANTOINETTE TORDESILLAS

LEV TRUSKINOVSKY

JUAN J. L. VELÁZQUEZ

VINCENZO VESPRI

ANGELO VULPIANI msp.org/memocs

Università di Roma “La Sapienza”, Italia

Rutgers University, USA

(CO-CHAIR) Università di Roma "La Sapienza", Italia

(TREASURER) Università dell'Aquila, Italia

University of California at Davis, USA

(CO-CHAIR) Université Paris-Nord, France

Università dell' Aquila, Italy

École Polytechnique, France

DAMTP Cambridge, UK, and University of Vienna, Austria

(CHAIR MANAGING EDITOR) Univ. of Illinois at Urbana-Champaign, USA

Université du Sud Toulon-Var, France

University of California at Berkeley, USA

Universität Erlangen-Nürnberg, Germany

LMA CNRS Marseille, France

Università di Roma “La Sapienza”, Italia

Università dell' Aquila, Italy

Université de Lyon-INSA (Institut National des Sciences Appliquées), France (CHAIR MANAGING EDITOR) Univ. of Illinois at Urbana-Champaign, USA

Carnegie Mellon University, USA, and Bilkent University, Turkey

Otto-von-Guericke-Universität Magdeburg, Germany

Università di Roma "La Sapienza”, Italia

University of Sheffield, UK

University of Novi Sad, Serbia

Wayne State University, USA

Université du Sud Toulon-Var, France

Università di Roma Tor Vergata, Italia

University of North Carolina at Chapel Hill, USA

Università di Pavia, Italia

Stanford University, USA

Institut Polytechnique de Grenoble, France

Università dell'Aquila, Italia

Università di Ferrara and International Research Center MEMOCS, Italia

Vanderbilt University, USA

Freie Universität Berlin, Germany

University of Texas at Austin, USA

Université Aix-Marseille, France

Cornell University, USA

École des Mines, France

University of Western Ontario, Canada

Università dell' Aquila, Italy

Louisiana State University, USA

Università dell' Aquila, Italia

Université de Lyon-INSA (Institut National des Sciences Appliquées), France

University of Pittsburgh, USA

Università di Roma "La Sapienza", Italia

Université Paris VI, France

Istituto per le Applicazioni del Calcolo "M. Picone", Italy

Universität Duisburg-Essen, Germany

Narvik University College, Norway, Russia

Università di Roma Tor Vergata, Italy

Università di Roma "La Sapienza", Italia

Università di Roma “Tor Vergata", Italia

Universidad Rey Juan Carlos, Madrid, Spain

McGill University, Canada

Moscow State Lomonosov University, Russia

Academy of Sciences of the Czech Republic

Universität zu Köln, Germany

University of Melbourne, Australia

École Polytechnique, France

Bonn University, Germany

Università di Firenze, Italia

Università di Roma La Sapienza, Italia

MEMOCS (ISSN 2325-3444 electronic, 2326-7186 printed) is a journal of the International Research Center for the Mathematics and Mechanics of Complex Systems at the Università dell'Aquila, Italy.

Cover image: "Tangle" by @ John Horigan; produced using the Context Free program (contextfreeart.org).

PUBLISHED BY

mathematical sciences publishers

nonprofit scientific publishing

http://msp.org/

(C) 2015 Mathematical Sciences Publishers 
Mathematics and Mechanics of Complex Systems vol. 3 no. 1

Effects of damping on the stability of the compressed

Nicolai beam

Angelo Luongo, Manuel Ferretti and Alexander P. Seyranian

Responses of first-order dynamical systems to Matérn, Cauchy, and Dagum excitations

Lihua Shen, Martin Ostoja-Starzewski and Emilio Porcu

Reflections on mathematical models of deformation waves in elastic microstructured solids

Jüri Engelbrecht and Arkadi Berezovski

On the approximation theorem for structured deformations from $B V(\Omega)$

Miroslav Šilhavý

MEMOCS is a journal of the International Research Center for the Mathematics and Mechanics of Complex Systems at the Università dell' Aquila, Italy.

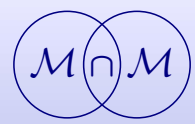

